\title{
The Protective Effect of Minocycline in a Paraquat-Induced Parkinson's Disease Model in Drosophila is Modified in Altered Genetic Backgrounds
}

\author{
Arati A. Inamdar, ${ }^{1,2}$ Anathbandhu Chaudhuri, ${ }^{1,3}$ and Janis O'Donnell ${ }^{1}$ \\ ${ }^{1}$ Department of Biological Sciences, University of Alabama, Tuscaloosa, AL 35487-0344, USA \\ ${ }^{2}$ Department of Plant Biology and Pathology, Rutgers University-The State University of New Jersey, Room 291D, Foran Hall, \\ 59 Dudley Road, New Brunswick, NJ 08901, USA \\ ${ }^{3}$ Department of Pharmacology and Experimental Neuroscience, University of Nebraska Medical Center, Omaha, \\ NE 68198-5800, USA \\ Correspondence should be addressed to Arati A. Inamdar, inamdar@rci.rutgers.edu
}

Received 10 January 2012; Accepted 4 June 2012

Academic Editor: Katerina Venderova

Copyright () 2012 Arati A. Inamdar et al. This is an open access article distributed under the Creative Commons Attribution License, which permits unrestricted use, distribution, and reproduction in any medium, provided the original work is properly cited.

\begin{abstract}
Epidemiological studies link the herbicide paraquat to increased incidence of Parkinson's disease (PD). We previously reported that Drosophila exposed to paraquat recapitulate PD symptoms, including region-specific degeneration of dopaminergic neurons. Minocycline, a tetracycline derivative, exerts ameliorative effects in neurodegenerative disease models, including Drosophila. We investigated whether our environmental toxin-based PD model could contribute to an understanding of cellular and genetic mechanisms of minocycline action and whether we could assess potential interference with these drug effects in altered genetic backgrounds. Cofeeding of minocycline with paraquat prolonged survival, rescued mobility defects, blocked generation of reactive oxygen species, and extended dopaminergic neuron survival, as has been reported previously for a genetic model of PD in Drosophila. We then extended this study to identify potential interactions of minocycline with genes regulating dopamine homeostasis that might modify protection against paraquat and found that deficits in GTP cyclohydrolase adversely affect minocycline rescue. We further performed genetic studies to identify signaling pathways that are necessary for minocycline protection against paraquat toxicity and found that mutations in the Drosophila genes that encode c-Jun N-terminal kinase (JNK) and Akt/Protein kinase B block minocycline rescue.
\end{abstract}

\section{Introduction}

The pathogenic mechanisms of PD, whether triggered by mutations or aberrant copy number of a PD-associated gene or by environmental sources, are associated with increased oxidative stress within dopaminergic neurons, exacerbated by the highly reactive nature of dopamine itself [1-3]. Chronic neuronal dysfunction overstimulates the normally protective neuroinflammatory response further amplifies oxidative conditions, accelerating disease progression $[4,5]$. Therefore, compounds with antioxidant and anti-inflammatory capabilities are of great interest for their potential therapeutic benefits in chronic neurodegenerative diseases including PD. Minocycline (MC), a second generation tetracycline drug known to be clinically safe [6], has shown promising ameliorative effects in animal models for chronic neurodegenerative diseases, including neurotoxin-induced PD models [7-9]. MC appears to have anti-inflammatory properties that mediate neuroprotection in PD animal models [10] as well as antioxidant properties [11]. Despite numerous reports of beneficial effects, however, other studies find deleterious consequences for MC treatment in some models of neurodegenerative disease and neuronal injury $[12,13]$ and damage to isolated mitochondria in micromolar concentrations [14]. Moreover, no single mode of action nor direct target of this antibiotic has been identified, which may partially account for the diversity of reported effects [15]. Genetic background is another 
feature that may contribute to these seemingly contradictory responses, as genetic variation amongst individuals is known to modify the functional activity of drugs [16]. Such genedrug interactions are cumbersome to investigate in humans or even in vertebrate model organisms. Invertebrate genetic models offer a simplified means of investigating three-way interactions between genes relevant to disease susceptibility and progression, environmental conditions, and therapeutic agents.

The genetic model organism, Drosophila melanogaster, is increasingly recognized as a useful model for neurodegenerative diseases due to its relative simplicity, ease of manipulation, high degree of conservation of neural mechanisms and signaling pathways, and availability of powerful genetic and molecular reagents for investigations of disease mechanisms $[17,18]$. Thus, this model system should facilitate dissection of relevant response pathways during disease progression and identification of those alter responses to drugs such as MC.

The effects of MC have been investigated mainly in mammalian models, although it has been shown that $\mathrm{MC}$ enhances survival of paraquat- (PQ-) fed Drosophila [19] and delays dopamine loss in a $D J-1$ genetic PD model in Drosophila [20]. We have developed a Drosophila model based upon ingestion of PQ, which recapitulates characteristic symptoms of PD with degeneration of dopaminergic neurons and accompanying neurological symptoms, including resting tremors and postural instability. Moreover, we demonstrated that mutations directly altering the regulation of DA homeostasis dramatically alter susceptibility to PQ $[21,22]$. Thus, this model offers the ability to simultaneously modify genetic background and environmental toxins.

In this paper, we confirm the results of previous studies $[19,20]$ utilizing this environmental toxin model to demonstrate that MC prolongs survival of PQ-exposed adult Drosophila and that it diminishes PQ-induced mobility defects, blocks associated changes in the DA homeostasis pathway, diminishes levels of reactive oxygen species (ROS), and prolongs DA neuron survival, as reported in the Drosophila DJ-1 model [20]. Moreover, we demonstrate that MC dose can dramatically alter the outcome of survival studies. We then extend the analysis of MC action by demonstrating that mutations in genes altering DA homeostasis and PQ susceptibility can affect the ameliorative action of MC. We further employ this system to identify signaling pathways that can modify PQ-induced toxicity and the protective effects of MC in this in vivo Drosophila model. Using loss of function mutants and overexpression transgenic lines, we found that JNK and Aktl play an important role in protecting DA neurons against PQ toxicity and reductions in expression of these kinases diminishes the ability of MC to protect against $\mathrm{PQ}$.

\section{Materials and Methods}

Drosophila Strains and Culture Maintenance. Two strains were utilized as wild type control lines in all experiments testing mutant strains: Canton $\mathrm{S}$, a wild type strain, and $y w^{1118}$, a yellow-body, white-eye strain that is otherwise wild type. For all experiments employing Gal4 expression to drive UAS-transgenes, TH-Gal4/+, and UAS-transgene/+ were utilized as controls. Mutant strains obtained from the Bloomington Drosophila Stock Center were as follows: rolled $\left(r l^{1}\right)$, a weak loss-of-function allele of the gene encoding ERK, $b s k^{1} / C y O$ and $b s k^{2} / C y O$ and $D f(2 L) J 27, b s k^{[J 27]} / C y O, P\{r y[+t 7.2]=\operatorname{sevRas} 1 . V 12\} F K 1$ loss-of-function alleles of the gene encoding JNK, $r y^{506} P\{P Z\} A k t 1^{04226} / T M 3, r y^{R K} S b^{1} \operatorname{Ser}^{1}$ and $y^{1} w^{67 c 23}$; $P\left\{w^{+m C} y^{+m D i n t 2}=E P g y 2\right\} A k t 1^{E Y 10012} / T M 3, S^{1} S^{1}$ carrying loss-of-function alleles of $A k t / P K B$, a loss-offunction allele for the gene encoding reaper, reaper, $y^{1}$ $w^{67 c 23} ; P\{S U P o r-P\} K G 07184 r y^{506}$, and a null mutant allele of Nedd2-like caspase (Nc/Dronc), $y^{1} w^{*} ; N c^{51} / T M 3, S b^{1}$. Transgenic strains employed for wild type expression of kinases were JNK (bsk), $y^{1} w^{1118} ; P\{U A S-b s k . A-Y\} 1$ and Akt1, $y^{1} w^{1118} ; P\{U A S-A k t 1\} / C y O$.

The transgenic strain UAS-2X eGFP (Chromosome II) was obtained from the Bloomington Drosophila Stock Center and a TH-Gal4 strain [23] was obtained from Jay Hirsh (University of Virginia). Loss-of-function Catsup alleles are described in Stathakis et al. [24], pale $e^{2}$ TM3, Sb is a loss-of-function mutation in the gene encoding $\mathrm{TH}$ [25]. The loss of function $P u^{Z 22}$ is described in Mackay et al. [26]. All mutants and transgenic strains were mated to the appropriate wild type strain, and all assays were performed on mutants heterozygous for the wild type genes. All stocks were maintained at $25^{\circ} \mathrm{C}$.

2.1. Feeding Experiments. Separated male and female flies, 48-96 hr after-eclosion, were fed on filter paper saturated with one of the following solutions: $5 \%$ sucrose, $5 \%$ sucrose with 1 or $10 \mathrm{mM}$ paraquat, minocycline $\mathrm{HCl}$ at varying concentrations, $10 \mathrm{mM}$ PQ with $1 \mathrm{mM} \mathrm{MC}$, and $1 \mathrm{mM}$ PQ with $200 \mu \mathrm{M}$ NG-nitro-L-arginine methylester (L-NAME). Feedings were continued as indicated in Section 3. All chemicals were obtained from Sigma (St. Louis, MO).

2.2. Locomotion Assay. The mobility of adult male and female flies from each treatment group was assessed using a negative geotaxis climbing assay. A single fly was placed in an empty plastic vial, tapped to the bottom, and the time required to climb $5 \mathrm{~cm}$ was recorded three times sequentially with 10 min rest periods between each measurement. Each replication value recorded was an average of the three trials; each assay was conducted on 10 flies per test group.

2.3. HPLC Analysis. Monoamine and pteridine levels were determined using an ESA CoulArray Model 5600A high performance liquid chromatography system. Fifty adult heads were extracted in $60 \mu \mathrm{L} 0.1 \mathrm{M}$ perchloric acid, followed by centrifugation. Ten $\mu \mathrm{L}$ aliquots of each extract were injected. Analyses were conducted on three replicas of each test set. Amines and pteridines were separated on a Phenomenex Synergi $4 \mu \mathrm{m}$ Hydro-RP column $(4.5 \times 150 \mathrm{~mm})$ according to the method of McClung and Hirsh [27]. Separations were performed with isocratic flow at $1 \mathrm{~mL} / \mathrm{min}$. Amines 
were detected with the ESA CoulArray electrochemical analytical cell, Model 5011 (channel 1 at $-50 \mathrm{mV}$, channel 2 at $300 \mathrm{mV}$ ). Pteridines were detected with a Linear Model LC305 fluorescence detector (excitation wavelength $360 \mathrm{~nm}$ and emission wavelength $456 \mathrm{~nm}$ ). Analysis was performed using ESA CoulArray software.

2.4. GTPCH Assay. GTPCH activity was assayed as previously described [21]. Briefly, extracts were prepared from 30 heads of 3-5 days old adult males in $100 \mu \mathrm{L} 50 \mathrm{mM}$ Tris, $2.5 \mathrm{mM}$ EDTA, and $\mathrm{pH}$ 8.0. Extracts were centrifuged at $10,000 \mathrm{rpm}$ for $10 \mathrm{~min}$ and the protein concentrations of supernatants were determined using the BioRad Protein Assay Reagent. GTP was added to extract corresponding to $45 \mu \mathrm{g}$ of protein to a final concentration of $0.2 \mathrm{mM}$ in a final volume of $70 \mu \mathrm{L}$. The mixture was incubated for $1 \mathrm{hr}$ at $37^{\circ} \mathrm{C}$ to convert GTP to dihydroneopterin triphosphate (dNP3), followed by its oxidation in $30 \mu \mathrm{L}$ of $1 \%$ iodine and $2 \%$ potassium iodide in $1 \mathrm{M} \mathrm{HCl}$ and dephosphorylation with 2 units of alkaline phosphatase (Roche). Neopterin peaks were detected by fluorescence at excitation wavelength of $353 \mathrm{~nm}$ and emission wavelength of $438 \mathrm{~nm}$.

2.5. Confocal Microscopy. Whole mounts of dissected brains from TH-Gal4; UAS-eGFP, TH-Gal4; UAS-eGFP/UAS$b s k^{W T}$, and TH-Gal4; UAS-eGFP/UAS-Akt ${ }^{W T}$ adults fed with sucrose alone, with paraquat, or with paraquat and minocycline together were examined for dopaminergic neuron morphology and number, detected by visualizing GFPexpressing neurons. Each brain was scanned to include 1518 sections for optimum visualization of DA neurons. The Z-sections were then utilized to get the average of all sections using a Leica TCS SP2 AOBS confocal microscope except for confocal images in Figure 8, which were captured using Zeiss LSM 710 Confocal Microscopy.

2.6. Catalase Assay. Crude enzyme extracts from adult flies fed $\mathrm{PQ}$ and $\mathrm{MC}$ as described above were prepared from ten heads from each treatment group in $150 \mu \mathrm{L} 0.1 \mathrm{M}$ sodiumpotassium phosphate buffer containing $0.1 \mathrm{M}$ Triton X-100 $(\mathrm{pH} 7.0)$, and activity assays were conducted following the method of [28]. The reaction of head extract with $\mathrm{H}_{2} \mathrm{O}_{2}$ was determined at absorbance wavelength $230 \mathrm{~nm}$ and calculated using a molar extinction coefficient for $\mathrm{H}_{2} \mathrm{O}_{2}$ of 62.4. One unit of catalase activity was defined as $1 \mu$ mole of $\mathrm{H}_{2} \mathrm{O}_{2}$ decomposed per min. All values represent the average of 6-8 replications from independently prepared extracts.

2.7. Lipid Peroxidation Assay. Head extracts from fifty heads were prepared in $100 \mu \mathrm{L}$ of $0.1 \mathrm{M}$ phosphate buffer from female flies at 2-4 days after eclosion fed as described above for $24 \mathrm{hr}$. Two $\mathrm{mL}$ of reagent TCA-TBA (thiobarbituric acid)$\mathrm{HCl}$ was added to $1 \mathrm{~mL}$ of head extract and heated for $15 \mathrm{~min}$ in a boiling water bath to allow malondialdehyde, the product of the lipid peroxidase reaction, to develop a red chromophore, detected spectrophotometrically at $535 \mathrm{~nm}$, as described by [29].
2.8. Griess Assay for NOS Activity. Head extracts from fifty heads were prepared in $100 \mu \mathrm{L}$ of $0.1 \mathrm{M}$ phosphate buffer with $1 \mathrm{M} \mathrm{KCl}(\mathrm{pH}$ 7.4). Following centrifugation to remove debris, the supernatants were mixed with freshly prepared Modified Griess reagent (Sigma) in a volume ratio of $1: 1$. After a $15 \mathrm{~min}$ incubation period at room temperature in dark, nitrite levels were measured spectrophotometrically at $595 \mathrm{~nm}$, with concentrations of nitrite calculated against a silver nitrite-derived standard curve and data was presented as a concentration of nitrite generated by extracts of $50 \mathrm{fly}$ heads.

2.9. Statistical Analysis. One-way ANOVA with Dunnett's post-test or by two-tailed Student's $t$-test were used to analyze the data using GraphPad Prism (San Diego, CA). The figure legends describe the analyses of the data.

\section{Results}

3.1. Effect of Minocycline on Paraquat-Induced Truncation of Life-Span. Because MC toxicity has been reported in some mammalian disease models, we first tested a range of $\mathrm{MC}$ concentrations from $100 \mu \mathrm{M}$ to $50 \mathrm{mM}$ on non-PQtreated flies to assess potential deleterious effects. We found that ingestion of $5 \mathrm{mM} \mathrm{MC}$ or greater affected viability, while lower concentrations caused no observable toxicity (Figure 1(a)). The results of this test for adult males are shown in Figure 1(a); females gave comparable results (data not shown). All subsequent experiments utilized MC concentrations of $1 \mathrm{mM}$ or less to avoid drug-related toxicity.

We then asked whether concentrations of antibiotic at $1 \mathrm{mM}$ or lower could rescue the toxic effects of $10 \mathrm{mM}$ PQ (Figure 1(b)). When exposed to PQ alone, the average survival duration of adult males was approximately three days. Cofeeding of MC at concentrations of $500 \mu \mathrm{M}$ and below did not improve survival duration; however, cofeeding of $1 \mathrm{mM} \mathrm{MC}$ with PQ extended the average survival duration an additional $48 \mathrm{hr}$, from three to five days (Figures $1(\mathrm{~b})$ and 1(c)). Unless otherwise noted, all subsequent experiments were performed using $10 \mathrm{mM} P Q$ and $1 \mathrm{mM}$ MC. We then tested the efficacy of MC under three different regimens, comparing $\mathrm{PQ}$ and $\mathrm{MC}$ co-feeding, prefeeding $\mathrm{MC}$ for 2 days prior to PQ exposure, and prefeeding PQ for 2 days prior to exposure to $\mathrm{MC}$ alone. We found that neither prefeeding nor posttreatment of $\mathrm{MC}$ were able to modify the survival duration of PQ-exposed flies (data not shown). However, extending the MC pre-feeding period to 5 days resulted in extension of life span to almost the same degree as the co-feeding regimen (Figure 1(c)).

3.2. MC Protects against PQ-Induced Mobility Defects. We employed a climbing assay, which is a sensitive indicator of the onset of dopaminergic neuron-linked movement dysfunction, to assess whether MC could rescue the mobility deficits induced by $\mathrm{PQ}$, similar to those observed by Faust et al. [20], in a Drosophila DJ-1 genetic model of PD and in several mammalian models [30,31]. Within $24 \mathrm{hr}$ of the initiation of $\mathrm{PQ}$ feeding in the absence of $\mathrm{MC}$, 


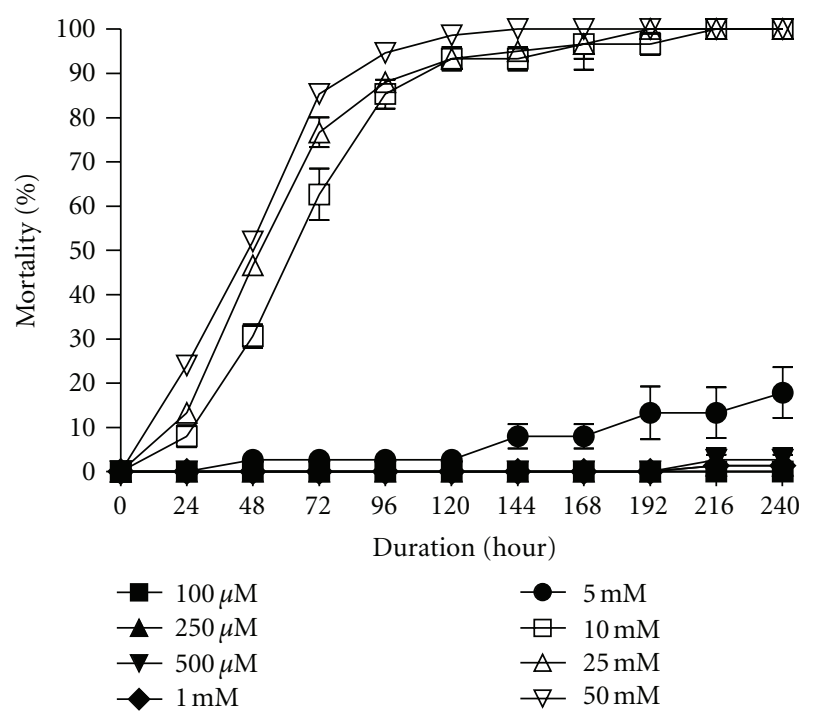

(a)

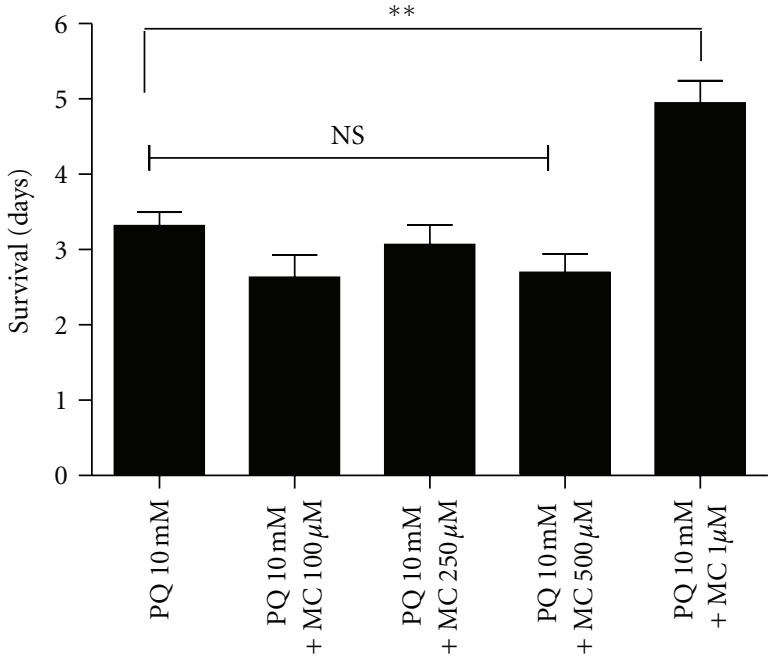

(b)

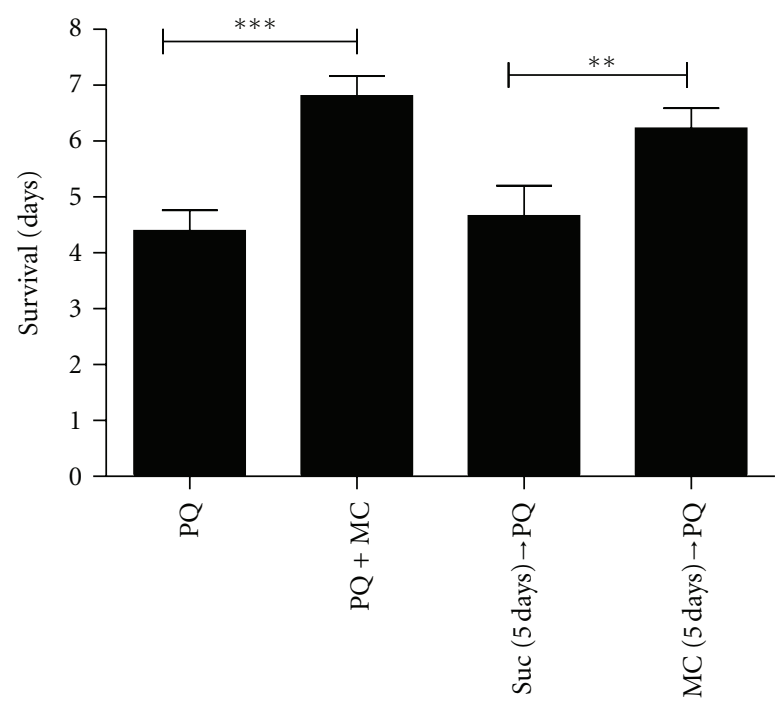

(c)

FIGURE 1: Effect of MC in absence and presence of $10 \mathrm{mM}$ PQ on survival of adult male flies. (a) Effect of increasing concentrations of MC $(100 \mu \mathrm{M}-50 \mathrm{mM})$ on survival of adult male flies. Data were collected every $24 \mathrm{hrs}$ for each group until $100 \%$ mortality was noted with $50 \mathrm{mM}$ MC. (b) Effect of different doses of MC co-fed with $10 \mathrm{mM}$ PQ on the life span of wild type adult males. Co-feeding of $1 \mathrm{mM}$ MC with $10 \mathrm{mM}$ PQ significantly extends the survival duration compared with PQ alone. ** represents the difference between PQ-fed flies and those fed PQ with $1 \mathrm{mM} \mathrm{MC}$ at $P<0.005$. NS = nonsignificant. Error bars indicate the standard error of the mean. Each data point was derived at least 10 replications of 15 flies each. (c) Wild type flies at $48 \mathrm{hrs}$ after eclosion were fed PQ and/or MC using different regimens as shown in the graph. The MC co-feeding group was compared to the PQ alone group and the MC prefeeding group was compared with the same aged group prefed 5\% sucrose. Both co-feeding and prefeeding regimens improved the survival duration compared to PQ alone. ${ }^{* *}=P<0.005$ and ${ }^{* * *}=P<0.001$ and represent the significant difference between PQ and prefeeding and co-feeding groups. Error bars represent SEM, and $n=50-60$ flies for all groups in (a), (b), and (c).

tremors and bradykinesia were apparent. At $48 \mathrm{hr}$, these flies were unable to climb and exhibited a strong bradykinesialike behavior. In contrast, when PQ was cofed with MC, no movement defects were apparent, and mobility was comparable to the negative geotaxis activity of control and minocycline-only flies (Figure 2).
3.3. MC Delays PQ-Induced Loss of Dopaminergic Neurons. We previously established that the onset of movement dysfunction upon PQ ingestion coincides with the loss of region-specific subsets of dopaminergic neurons in the adult brain [21]. In light of the ability of MC to ameliorate PQ-induced tremors and mobility deficits, we next asked 


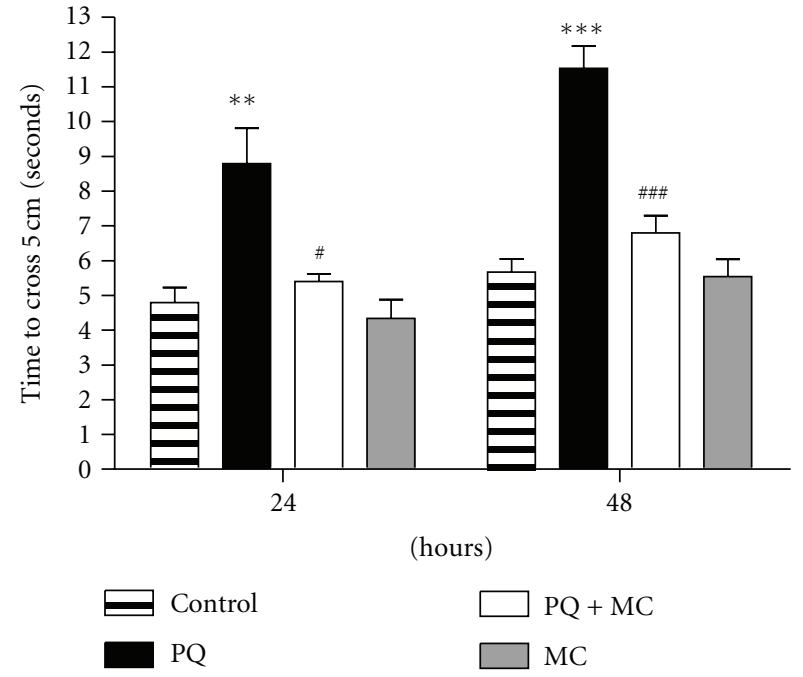

FIgUre 2: MC protects against PQ-induced mobility defects. The time required for adult male flies to climb $5 \mathrm{~cm}$, after 24 and $48 \mathrm{hr}$ of exposure to $10 \mathrm{mM}$ PQ or $10 \mathrm{mM}$ PQ with $1 \mathrm{mM} \mathrm{MC}$ flies was assayed. The ingestion of $1 \mathrm{mM} \mathrm{MC}$ alone has no effect on mobility, while ingestion of PQ alone adversely affects mobility. PQ and MC co-feeding results in mobility performance near control levels. The * and ${ }^{\#}$ indicate the significance of differences between control and PQ-fed flies and between PQ only and co-fed flies, respectively.

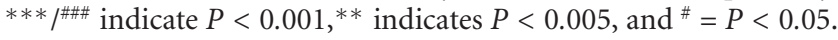
Error bars represent standard error of the mean. Each data point represents at least 15 replications of 10 flies each.

whether this effect is mediated through protection of atrisk dopaminergic neurons. Dopaminergic neurons were detected by the TH promoter-directed expression of GFP in the transgenic strain, TH-Gal4; UAS-eGFP. We compared dopaminergic neuron morphology and numbers in brains at 24 and $48 \mathrm{hr}$ after the initiation of feeding $5 \%$ sucrose only, PQ only, MC only, or PQ with MC (Figure 3). Figure 3(a) displays characteristic results in dopaminergic neuron cluster PPM2 after $24 \mathrm{hr}$ of treatment. The number and morphology of these neurons after the MC-only treatment were indistinguishable from neurons in control brains. As we had observed previously, using both the GFP reporter and immunolocalization of $\mathrm{TH}$ to identify dopaminergic neurons [21], these neurons display characteristic patterns of morphological changes and neuronal sensitivity after PQ exposure (Figure 3(a)), whereas neurons in animals exposed to $\mathrm{MC}$ with $\mathrm{PQ}$ retained near normal morphology and neuron number (Figures 3(a), 3(b), and 3(c)). Upon treatment with PQ only (Figure 3(a)) for $24 \mathrm{hrs}$, we observed that the PAL subgroup in the anterior region and the PPL1, PPM2, and PPM3 subgroups in the posterior brain exhibited statistically significant neuron loss relative to controls (Figure 3(b)). In the animals that were co-fed PQ and MC, there was no neuron loss in the PPM1 or PPL2 subgroups at $24 \mathrm{hr}$, and neuron numbers and morphology in other clusters were almost identical to those in control brains (Figures 3(a) and 3(b)). After 48 hrs of PQ feeding, previously affected clusters continued to deteriorate, while neurodegeneration was noted in the previously unaffected PPM1 and PPL2 clusters, while improved survival of all dopaminergic neuron groups was noted in the PQ and MC co-fed flies (Figure 3(c)). Therefore, we conclude that the extension of life span observed when flies were fed MC along with PQ and delay of the onset of movement deficits correlate with protection against $\mathrm{PQ}$-induced neurodegeneration.

3.4. MC Blocks Changes in DA Pathway Components Indicative of PQ-Induced Oxidative Stress. The production of DA is rate-limited by two enzymes: tyrosine hydroxylase (TH), which converts tyrosine to L-DOPA, and GTP cyclohydrolase (GTPCH), which is rate-limiting for the production of tetrahydrobiopterin $\left(\mathrm{BH}_{4}\right)$, a cofactor for and regulator of $\mathrm{TH}$ catalysis. We previously observed that sensitivity to $\mathrm{PQ}$ is at least partially defined by the activity of the $\mathrm{BH}_{4}$ and DA biosynthesis pathways [21]. We found that following PQ ingestion, but prior to loss of dopaminergic neurons, there is a transient stimulation of DA pathway activity, followed by a decrease in $\mathrm{BH}_{4}$ and DA levels and a corresponding increase in oxidative products, biopterin and DOPAC, respectively. The observed protection by minocycline against the effects of PQ might be mediated through interactions with the DA homeostasis machinery, either modulating DA synthesis or transport or through its ability to act as a scavenger of ROS [11], delaying the PQ-induced oxidative depletion of DA and subsequent oxidative damage. Ingestion of $\mathrm{MC}$ alone for $24 \mathrm{hr}$ has no significant effect on the production of pathway metabolites or on GTPCH activity (Figure 4), ruling out the possibility that MC modulates DA homeostasis. PQ induced dynamic changes of enzyme activity, pathway products, and oxidative products. After $24 \mathrm{hr}$ of PQ exposure, L-DOPA pools are significantly elevated, indicating an increase in $\mathrm{TH}$ activity; however, DA was depleted and the DA metabolite, DOPAC (3,4-dihydroxyphenylacetic acid) was elevated, as expected in an oxidative environment (Figures 4(a) and 4(b)). Similarly, GTPCH activity increased (Figure 4(c)), while $\mathrm{BH}_{4}$ pools are diminished and the oxidized product biopterin increased with PQ exposure (Figure 4(a)). In contrast, when MC was co-fed with PQ for $24 \mathrm{hr}$, the effect of $\mathrm{PQ}$ on each of these components was significantly less severe, consistent with the reported antioxidant property of MC.

3.5. MC Reduces PQ-Generated Reactive Oxygen Species. The ability of MC to block the PQ-induced changes in the $\mathrm{DA}$ and $\mathrm{BH}_{4}$ biosynthesis pathways indicative of oxidative stress led us to hypothesize that MC was serving principally as a scavenger of PQ-generated ROS in Drosophila. We employed two assays for oxidative stress, lipid peroxidation [29], and induction of catalase activity [28, 32]. PQ ingestion results in a two-fold elevation of lipid peroxidation within the first $24 \mathrm{hr}$ (Figure 5(a)). Co-feeding of MC with PQ almost completely blocks this indicator of lipid damage. Elevated catalase activities were detected in the heads of both PQ and PQ plus MC groups (Figure 5(b)); however, the catalase activity in flies that had ingested MCwith PQ was significantly lower than those exposed to PQ only. 

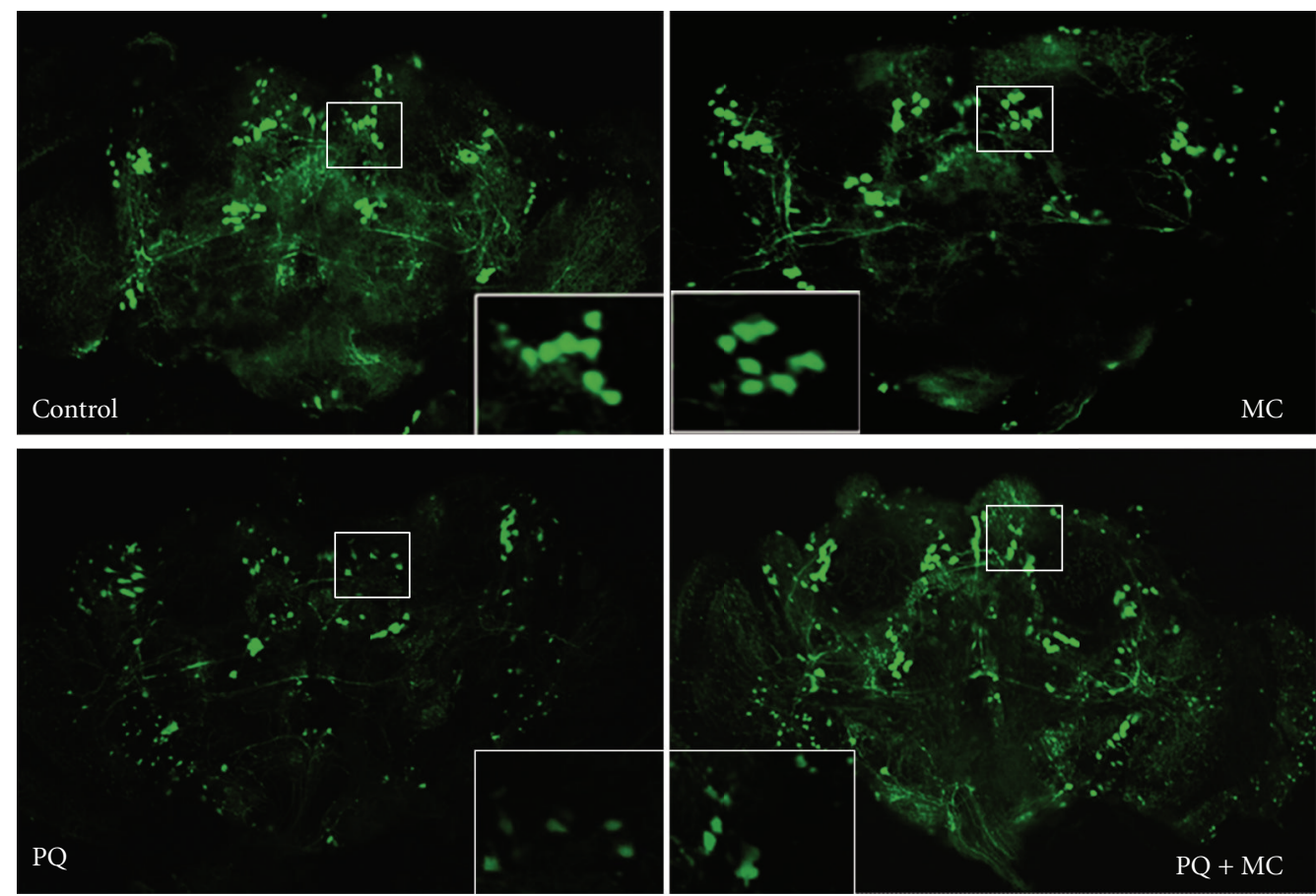

(a)
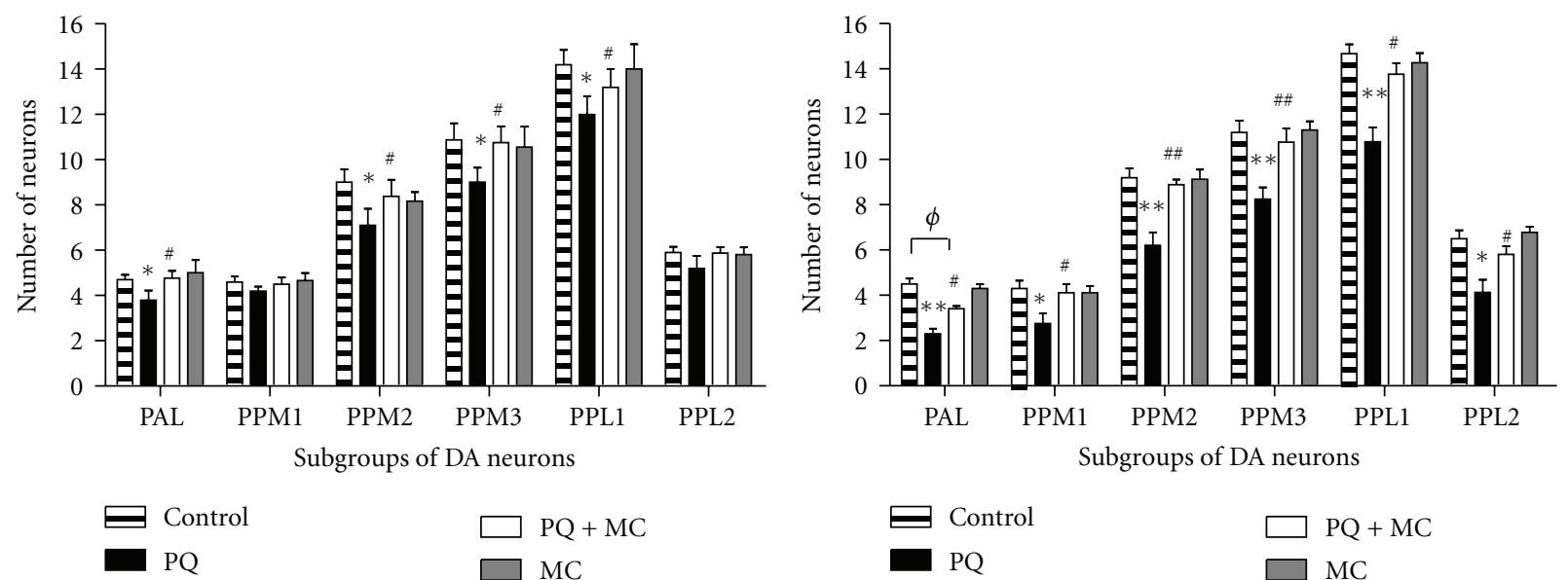

(b)

(c)

FIGURE 3: MC confers protection to dopaminergic neurons. (a) The effect of a $24 \mathrm{hr}$ exposure of adult males to sucrose (Control), $1 \mathrm{mM}$ MC alone (MC), $10 \mathrm{mM}$ PQ alone (PQ), and $10 \mathrm{mM}$ PQ with $1 \mathrm{mM} \mathrm{MC} \mathrm{(PQ} \mathrm{+} \mathrm{MC)} \mathrm{on} \mathrm{the} \mathrm{dopaminergic} \mathrm{neurons} \mathrm{of} \mathrm{TH-GAL4;} \mathrm{UAS-eGFP}$ adult brains. The inset in each image demonstrates the change in the morphology and number of the PPM2 subgroup of neurons. The exposure to $\mathrm{MC}$ alone $(\mathrm{MC})$ and sucrose (control) does not alter the number or morphology of the dopaminergic neurons. The addition of MC to PQ delays neuron loss and onset of abnormal neuron morphology relative to PQ only. Scale bar for whole brain images $=100 \mu \mathrm{m}$. (b) and (c) MC delays PQ-induced selective loss of dopaminergic neurons. The average number of neurons per subset was determined $24 \mathrm{hr}$ (b) and $48 \mathrm{hr}$ (c) after the initiation of feeding and shows that MC delays, but does not prevent, PQ-mediated neuronal loss in different dopaminergic neurons. Each subset of dopaminergic neurons was scored separately in 15-25 brains. The significance of the difference in each neuron cluster between the PQ-treated and control groups and between the PQ-treated and co-fed groups is indicated as * and ${ }^{\#}$, respectively, where ${ }^{*}=P<0.05,{ }^{* *}=P<0.005,{ }^{\#}=P<0.05$, and ${ }^{\# \#}=P<0.005$. ${ }^{\phi}$ represents the significant difference between control and $\mathrm{PQ}$ with MC co-fed brains where ${ }^{\phi}=P<0.05$. Error bars represent standard error of the mean.

These results strongly suggest that MC has a strong capacity to suppress ROS generated by PQ.

3.6. MC Cannot Rescue Pu (GTP Cyclohydrolase) Mutants. Mutations in the rate-limiting genes for $\mathrm{BH}_{4}$ and DA synthesis, Punch (Pu; GTPCH) [33] and pale (ple; TH) [25], respectively, result in decreased DA pools in adult heads and increased sensitivity to PQ [21]. Conversely, loss-of-function alleles of Catecholamines up (Catsup), a negative regulator of TH and GTPCH, have elevated $\mathrm{BH}_{4}$ and DA pools and 


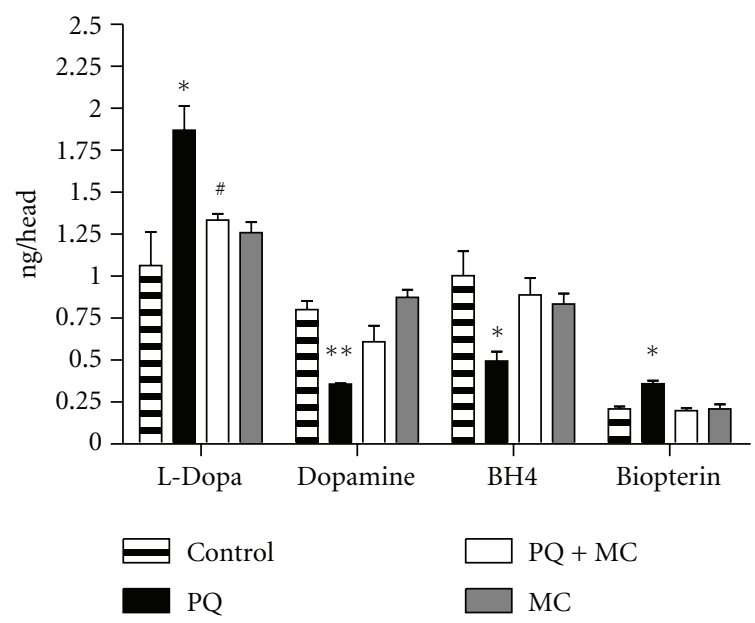

(a)

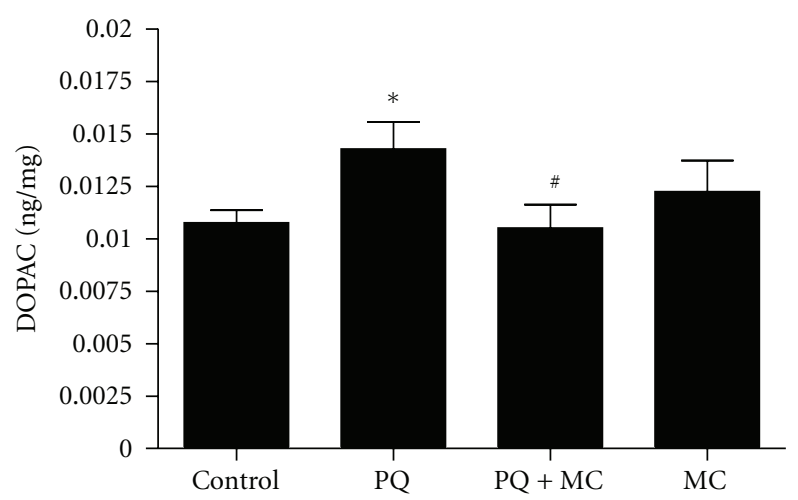

(b)

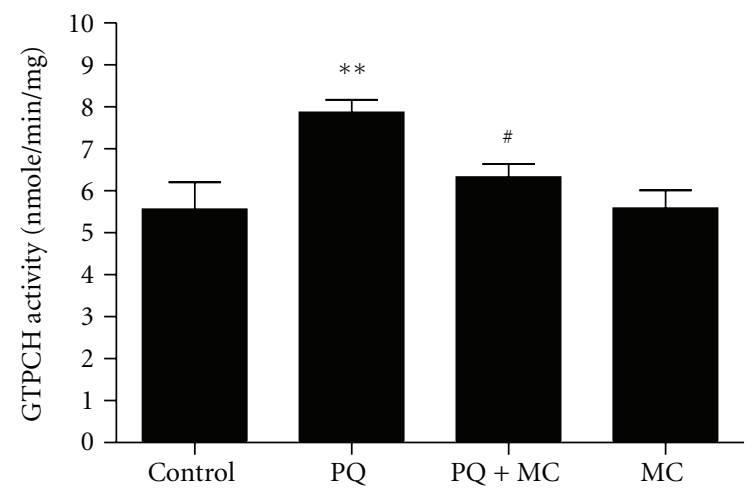

(c)

Figure 4: MC blocks the changes induced by PQ in the $\mathrm{DA}$ and $\mathrm{BH}_{4}$ biosynthesis pathways. (a) Changes in the $\mathrm{DA}$ and $\mathrm{BH}_{4}$ metabolites in adult males exposed to $10 \mathrm{mM}$ PQ or $10 \mathrm{mM}$ PQ with $1 \mathrm{mM} \mathrm{MC}$ for $24 \mathrm{hrs}$. The increase in L-DOPA levels, indicative of PQ-stimulated TH activity, is reduced by MC. (b) The DA metabolite, DOPAC, is elevated by PQ exposure and is significantly decreased by the co-feeding of MC to male adults for $24 \mathrm{hr}$. (c) The compensatory increase in GTPCH activity in PQ-fed adult males is reduced in PQ-MC co-fed males at $24 \mathrm{hr}$ of ingestion. The significance of differences in each subset between the PQ-treated and control groups, and PQ-treated and co-fed groups is indicated as ${ }^{*}$ and ${ }^{\#}$, respectively, where ${ }^{*}$ and ${ }^{\#}=P<0.05$ and ${ }^{* *}$ and ${ }^{\# \#}=P<0.005$. Error bars represent the standard error of the mean. Each data point represents at least 10 replications of 15 flies each.

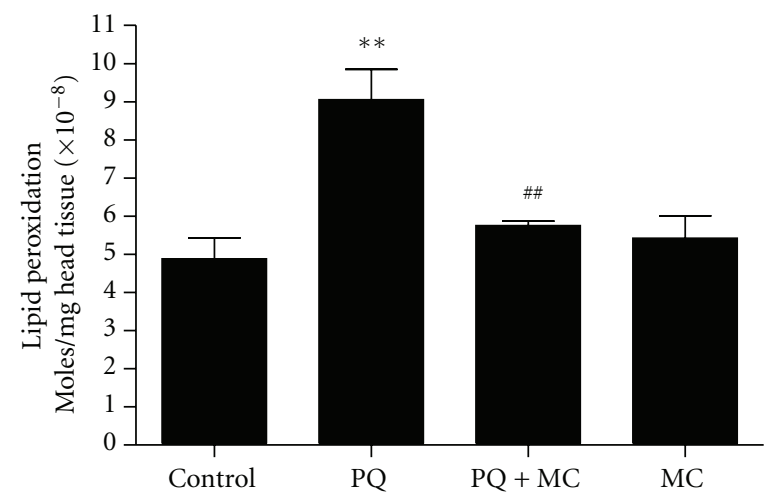

(a)

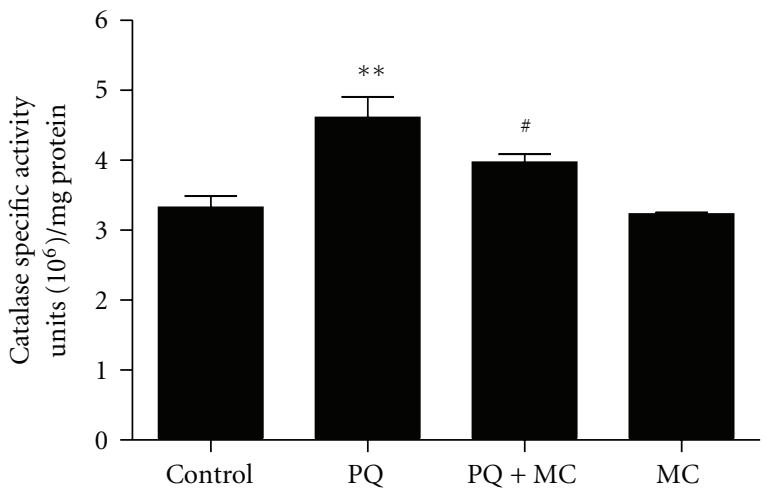

(b)

Figure 5: MC reduces PQ-generated reactive oxygen species. (a) The level of lipid peroxidation product was determined in head extracts of males that had ingested PQ or PQ with MC for $24 \mathrm{hr}$. MC significantly decreases the amount of lipid peroxidation induced by PQ. (b) MC reduces the specific activity of catalase after $24 \mathrm{hr}$ of ingestion in co-fed male flies. The significance of differences between the PQ-treated and control groups and between the PQ-treated and co-fed groups was indicated as ${ }^{*}$ and ${ }^{*}$, respectively, where ${ }^{*}$ and ${ }^{*}=P<0.05$. Error bars represent standard error of the mean. The experiments were done as 5-8 replicas of 10 head extracts. 
a strong resistance to $\mathrm{PQ}[22,24]$. While we continue exploring the mechanistic basis for the differential sensitivity to $\mathrm{PQ}$, these mutant strains provided the opportunity to begin defining genetic components that might have roles in modulating the protective effects of MC. We found that Catsup heterozygotes survive PQ 24 hrs longer, on average, than wild type adults, while $P u$ and ple heterozygotes die $48 \mathrm{hrs}$ or more before wild type flies (Figure 6(a)). MC extended the survival of wild type flies and the Catsup and ple mutants, by approximately 2 days for each strain. Therefore, we detected no DA-specific interactions with $\mathrm{MC}$ in these mutants.

Strikingly, however, MC was unable to improve the survival of $\mathrm{Pu}$ mutants under these conditions. One possibility is that the heterozygous $P u$ mutants might provide a sensitized background which reveals an otherwise undetectable deleterious effect of $1 \mathrm{mM}$ MC. However, in other studies, we found that ingestion of $\mathrm{MC}$ at concentrations up to $5 \mathrm{mM}$ by $P u$ mutants had no discernable effect on survival (Ajjuri and O'Donnell, in preparation). Alternatively, oxidative damage in $P u$ mutants, perhaps related to a $P u$ function other than regulation of DA synthesis, might progress too rapidly for $\mathrm{MC}$ to impart its protective effect. One such candidate is nitric oxide synthase (NOS), which requires $\mathrm{BH}_{4}$ as a cofactor. It is well known that limiting $\mathrm{BH}_{4}$, which should be a consequence of loss-of-function mutations in $\mathrm{Pu}$, results in the catalytic uncoupling of NOS, dramatically enhancing oxidative stress through production of elevated peroxides and peroxynitrites $[34,35]$. Since $P u$ mutants have reduced levels of $\mathrm{BH}_{4}$ [33], we hypothesized that the failure of MC protection in $\mathrm{Pu}$ mutants is linked to catastrophic oxidative damage stemming from induction of NOS by PQ and its subsequent uncoupling. If this hypothesis is correct, then inhibition of NOS catalytic activity should limit the production of ROS and RNS and, thereby, improve survival of the $P u$ mutant flies. As expected, heterozygous $P u$ mutants have lower NOS activity than wild type flies. Ongoing experiments to date have revealed no detectable effects of MC alone on NOS activity (unpublished data). PQ ingestion resulted in elevated NO production, as determined by the Griess assay, in both wild type and $P u$ mutant heads (Figure 6(b)). MC reduced nitrite levels in wild type flies, suggesting its ability to reduce the inflammatory response in flies as in mammals. However, MC was ineffective in reducing nitrite levels in $\mathrm{Pu}$ mutants, exposed to $10 \mathrm{mM}$ PQ (Figure 6(b)). We then fed $1 \mathrm{mM} \mathrm{PQ}$, to slow the rate of accumulation of oxidative damage, in an effort to further dissect events contributing to neurodegeneration. We observed that NOS inhibitor L-NAME reduced nitrite levels in both wild type and $\mathrm{Pu}$ mutants (Figure 6(c)). Moreover, ingestion of L-NAME was able to prolong survival of the $\mathrm{Pu}$ mutant strain as well as wild type flies exposed to $1 \mathrm{mM}$ PQ (Figure 6(d)). In contrast, even at this 10-fold lower concentration of $\mathrm{PQ}, \mathrm{MC}$ was still unable to improve the survival of $P u$ mutants. These results suggest that the failure of MC to rescue $P u$ mutants was due to its inability to limit NOS activity and therefore reduce oxidative damage when $\mathrm{BH}_{4}$ production is compromised.
3.7. Loss of Function Mutants of the Genes Encoding JNK and Akt Are Sensitive to $1 \mathrm{mM} P Q$ but Involvement of Reaper, Caspase, and Rolled in PQ-Induced Toxicity Was Not Detected. Signal transduction pathways associated with oxidative stress, inflammatory, and apoptotic responses are likely targets of minocycline action [15]. However, it also is known that dopaminergic signaling and homeostasis, highly conserved processes in flies and mammals, are highly responsive to environmental stressors across species lines [36, 37]. This feature of dopaminergic function is highlighted by our findings that PQ induces early changes in DA metabolism and that mutations in genes altering DA homeostasis strongly affect the sensitivity of Drosophila to $\mathrm{PQ}[21,22]$. The roles of such signal transduction pathways are likely to be highly complex, as discrepancies in evidence for participation of particular kinases in responses to oxidative damage and subsequent neurodegeneration emphasize [38, 39]. As a foundation towards employing our whole organism disease model to better understand the interface between regulation of signal transduction and dopamine homeostasis in dopaminergic neurodegeneration, we next turned our attention to MC effects under conditions in which specific signaling pathways previously implicated in neurodegeneration mechanisms are genetically modified.

The mitogen-activated protein kinase (MAPKs) subfamilies, extracellular signal regulated kinases (ERK), c-Jun Nterminal kinases (SAPK/JNK), and p38 kinases, are known to be activated by a wide range of stimuli including inflammatory cytokines and diverse environmental stressors and to mediate a variety of downstream effects likely to be integral to protection against neurodegenerative mechanisms [40, 41]. In mammalian models for neuronal injury and neurodegenerative diseases, pharmacological approaches have provided evidence that p38 and JNK are mainly implicated in neuronal death processes, while ERK may promote cellular recovery/survival from neuronal death implicated in these conditions [42].

We therefore tested heterozygous mutants for JNK, encoded by the gene basket and Akt/PKB, encoded by Akt1, and ERK, encoded by rolled, in Drosophila. In addition, we tested mutations in the caspase- 9 gene, dronc, and mutations in the proapoptotic gene, reaper. These kinases function in myriad biological processes but are particularly known to respond to various cellular stress. We hypothesized that they should also play key roles in the toxic responses triggered by PQ.

We exposed heterozygous mutants with loss of function alleles of $b s k$ and $A k t$ to $10 \mathrm{mM}$ PQ alone or in combination with $1 \mathrm{mM} \mathrm{MC}$ and observed that both sets of mutants had apparently slightly elevated sensitivity to PQ although not statistically significant (Figure 7(a)). Interestingly, however, MC failed to improve the survival of either mutant, while survival of the control strain was significantly improved. To clarify the effects of mutations in these kinase genes, we tested additional $b s k$ and $A k t$ alleles, decreasing the PQ concentration $(1 \mathrm{mM})$ to slow the progression of PQ-induced damage as in the Punch mutant experiments described above. In this experiment, we expanded our analysis to include a mutant allele of the ERK gene, rolled, and mutants for the 


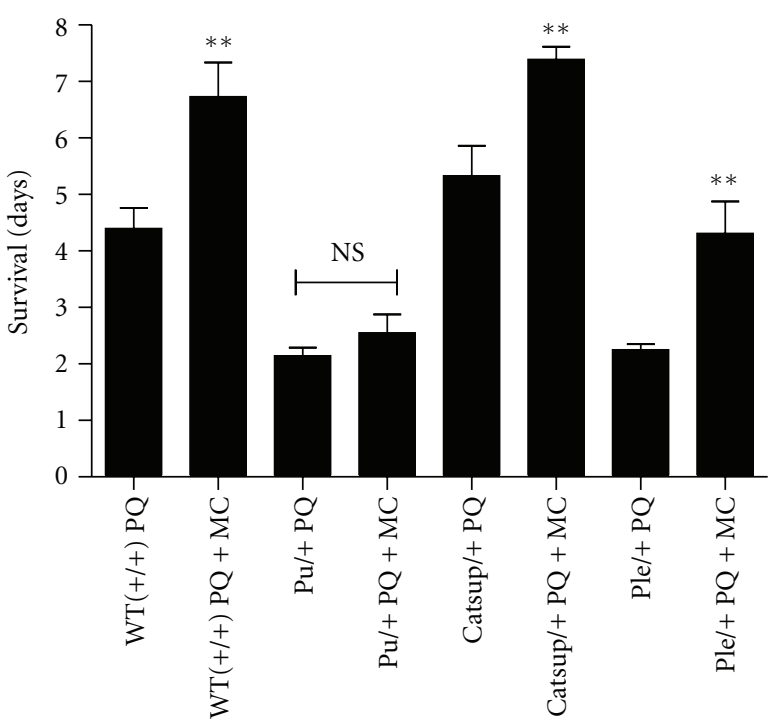

(a)

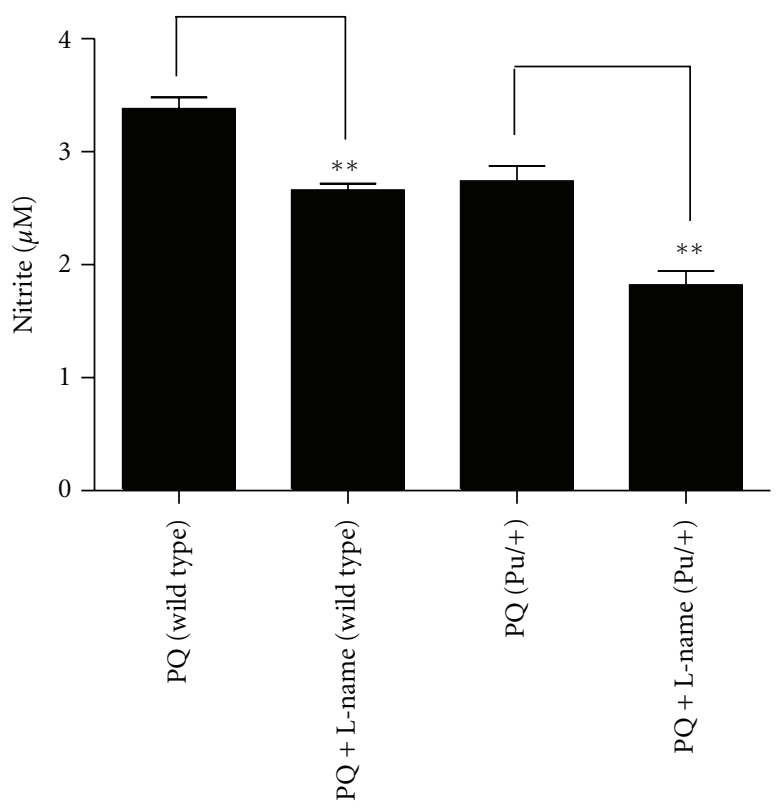

(c)

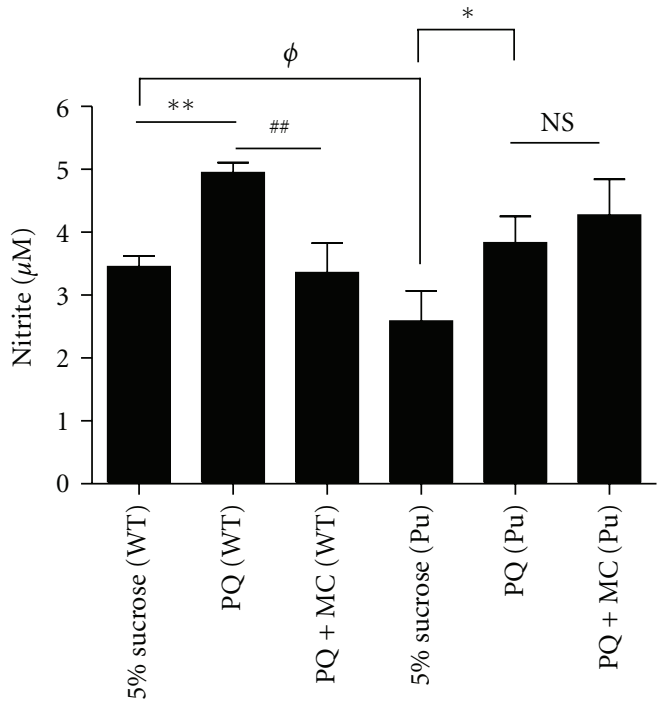

(b)

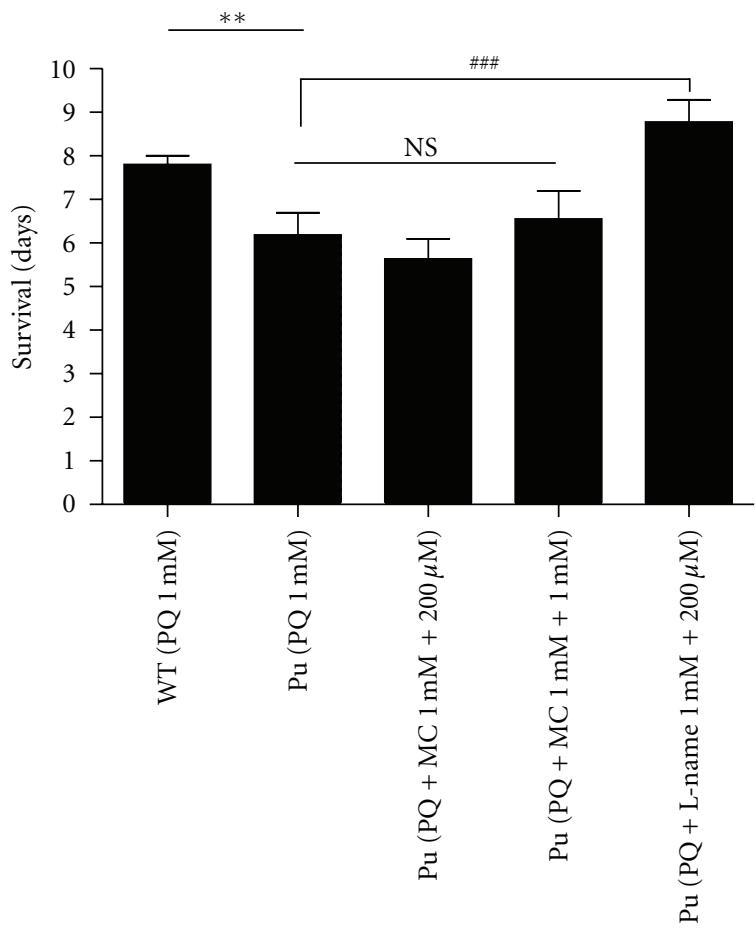

(d)

Figure 6: MC fails to rescue Punch mutants due to dysregulation of nitric oxide synthase catalytic function. (a) Effect of $1 \mathrm{mM}$ MC co-fed with $10 \mathrm{mM}$ PQ on DA regulatory mutants, Catsup $p^{26} /+, P u^{Z 22} /+$ and $p l e^{2} /+$. Catsup and ple mutant flies showed extension of life span, while $P u$ mutants did not. NS = not significant. ${ }^{* *}=P<0.005$ and represents significant differences between PQ and PQ with MC. (b) After $24 \mathrm{hr}$ of PQ, or PQ with MC exposure, suppression of NOS was detected in wild type heads but not in Punch mutants where NO levels of non-PQ-treated $P u$ mutants assayed are significantly lower than NO levels of non-PQ-treated wild type heads. ${ }^{*}$ or ${ }^{\phi} P=<0.05$ and ${ }^{* *}$ or ${ }^{\# \#} P=<0.005$. (c) Co-feeding of L-NAME with $1 \mathrm{mM}$ PQ reduced NO production in wild type and $P u$ mutants. ${ }^{* *}=P<0.005$. (d) The survival of $P u$ mutants was improved by co-feeding PQ with L-NAME, but not with MC when compared with survival of $P u$ mutants on PQ alone. * represents significant differences between control and PQ-exposed flies, while * represents significant differences between flies fed PQ only and PQ with MC. ${ }^{* *}=P<0.005$ and ${ }^{\# \#}=P<0.001$. NS $=$ not significant. Error bars represent standard error of the mean and $n=100-120$ fly heads for NOS assays and 50-60 flies for survival where experiments were replicated thrice with the same sample size. 


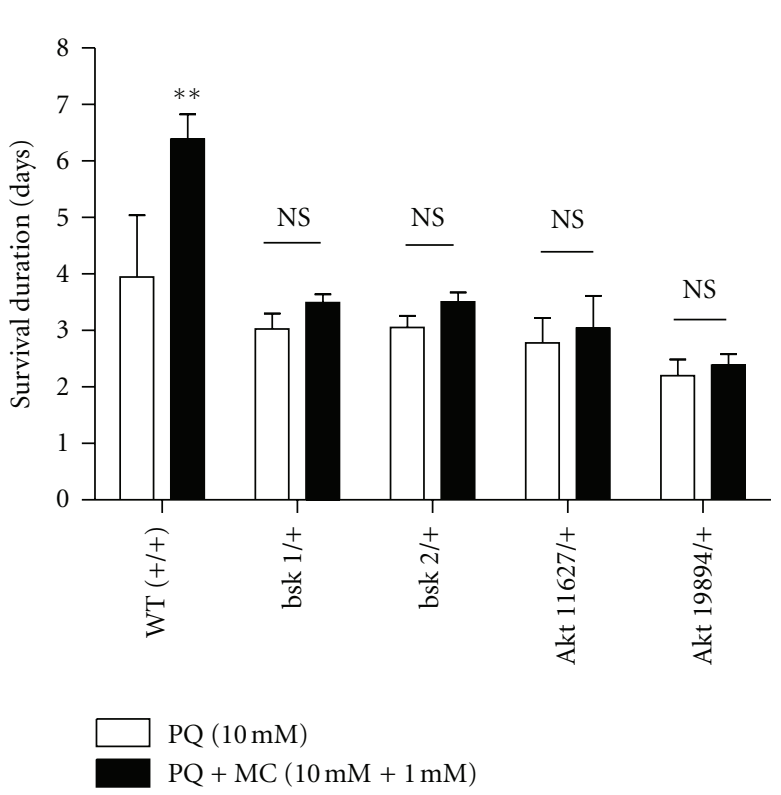

(a)

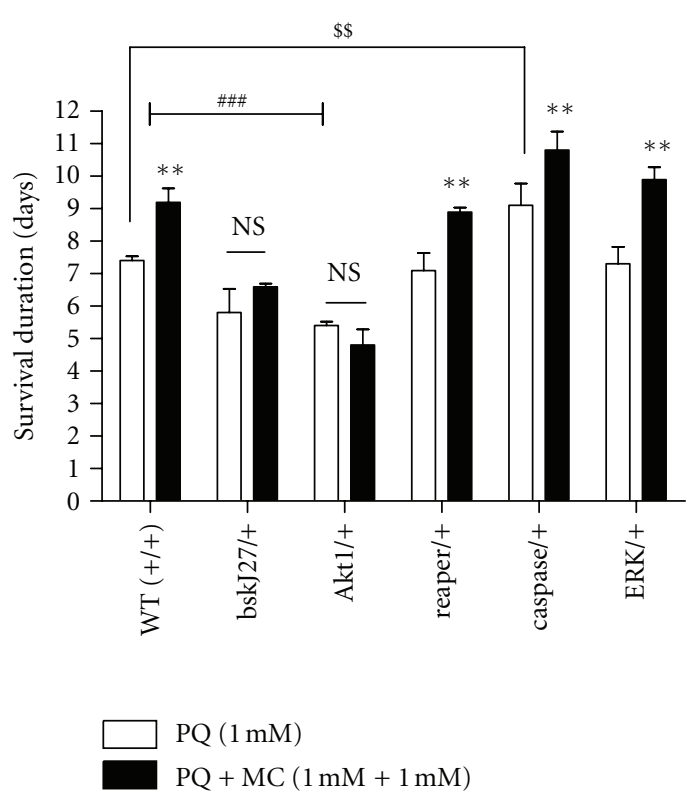

(b)

FIgURE 7: PQ and MC effects on viability are modified by components of stress response pathways. (a) Effect of $b s k$ and $A k t$ loss-of-function mutant alleles on PQ and MQ effects on survival. Treatment with $1 \mathrm{mM}$ MC failed to improve the survival of bsk and Akt loss-of-function mutants fed $10 \mathrm{mM}$ PQ. $n=120$ and each data point represents at least three independent replications of 40 flies for each.genotype. (b) Effect of $1 \mathrm{mM} P Q$ and $1 \mathrm{mM} \mathrm{MC}$ on loss-of-function mutants of signaling pathways, JNK, Akt, reaper, caspase, and ERK. rolled (ERK), reaper and caspase mutants showed an extension of life span with MC treatment, while the survival of JNK and Akt mutants was unmodified in the presence of MC. ${ }^{* *}=P<0.005$ represents significant difference between PQ and PQ with MC groups. ${ }^{\# \#}=P<0.005$ and shows a significant difference between PQ-fed wild type and PQ-fed $b s k$ and $A k t$ loss-of-function mutants. NS $=$ not significant. $\$ \$=P<0.005$ represents significant difference between PQ-fed wild type and PQ-fed caspase loss-of-function mutant. Error bars represent standard error of the mean. $n=180$ and each data point represents at least three independent replications of 50-60 flies each.

apoptotic pathway genes dronc (Caspase) and reaper. Under these conditions, the heterozygous bsk and Akt mutants showed increased sensitivity to $\mathrm{PQ}$, dying, on average, two days before wild type flies, and again MC was ineffective in rescuing these mutants (Figure $7(\mathrm{~b})$ ). These results suggest either that JNK and Akt signaling pathways are important in the protective response of $\mathrm{MC}$ in flies or that diminution of their activity heightens the oxidative environment to a point that the beneficial effects of MC are lost. However, the sensitivity of heterozygous reaper and rolled mutants to $\mathrm{PQ}$ and the ability of $\mathrm{MC}$ to increase survival were indistinguishable from the wild type controls. In contrast, the heterozygous caspase mutant, dronc, survived two days more than the wild type controls on PQ and minocycline improved survival an additional two days (Figure 7(b)).

\subsection{Overexpression of JNK and AKT Confers Protection} against Paraquat-Induced Toxicity in Dopaminergic Neurons. The observations that JNK and Akt loss-of-function heterozygous mutants increase the sensitivity to PQ and that normal expression of these genes appears to be important for MC-mediated protective responses against PQ led us to test whether this effect is reversed when JNK and Akt are overexpressed. Since PQ toxicity initially is observed in dopaminergic neurons and DA itself appears to interact in this process, we drove expression of wild type JNK and
Akt in DA neurons using the GAL4-UAS system [43]. Even though we employed PQ at the higher concentration of $10 \mathrm{mM}$ for these experiments, the expression of JNK and Akt in dopaminergic neurons resulted in approximately a two-fold increase in the survival duration in both cases (Figure 8(a)). MC improved life span in all strains by $30 \%$ compared to $10 \mathrm{mM}$ PQ alone. Importantly the combination of MC plus overexpression of Akt in dopaminergic neurons enhanced survival over three-fold relative to the wild type control. These results demonstrate that both Akt and JNK have prosurvival functions in PQ-induced DA toxicity, with Akt expression having the strongest effect.

We further verified the protective effect of wild type JNK and Akt by examining the survival of at-risk dopaminergic neurons in the adult brain. The transgenic strain, TH-Gal4; $U A S-e G F P$ was crossed with $U A S-J N K^{W T}$ and $U A S-A k t^{W T}$, expression of GFP in concert with JNK or Akt. We observed that $\mathrm{MC}$ was able to protect against neuron loss in subgroups of posterior brain DA neurons expressing GFP, but otherwise wild type, relative to the brains of flies from the same cultures exposed to PQ only (Figure 8(b)). The overexpression of Akt or JNK in dopaminergic neurons prevents PQ-induced DA neuron loss in most subgroups of DA neurons relative to the control brains. Similarly, the transgenic brains, TH-GAL4; UAS-eGFP/UAS-bsk ${ }^{W T}$ 


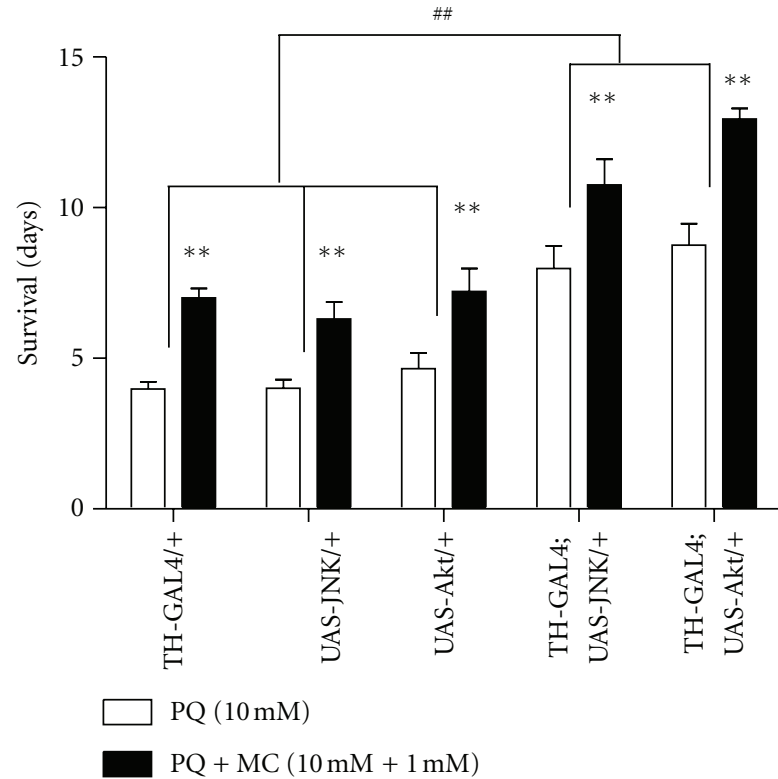

(a)

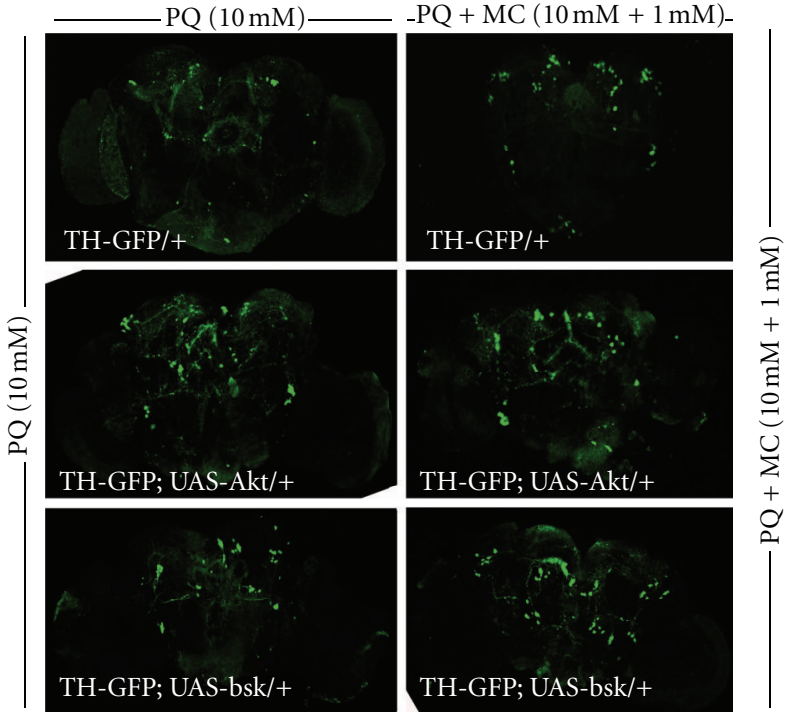

(b)

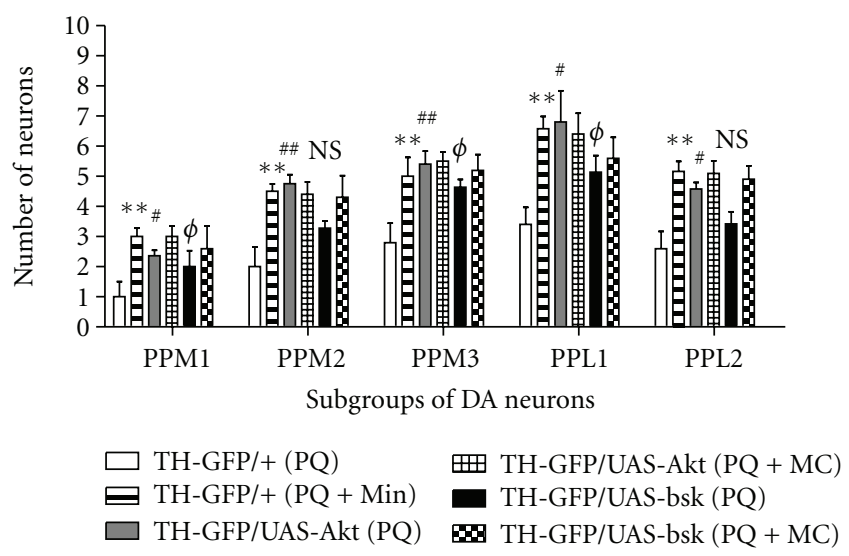

(c)

FIGURE 8: Overexpression of wild type BSK/JNK and Akt provides protection against PQ. (a) Adult males of genotypes TH-GAL4/+, $U A S-b s k^{1} /+, U A S-A k t^{1} /+, T H-G A L 4 ; U A S-b s k^{1} /+$, and TH-GAL4; UAS-Akt $t^{1} /+$ flies were fed, beginning at $48 \mathrm{hr}$ after eclosion, $10 \mathrm{mM}$ PQ or $10 \mathrm{mM}$ PQ and $1 \mathrm{mM}$ MC. The average survival duration for each group was determined. ${ }^{* *}=P<0.005$ and represents significant differences between the PQ and PQ with MC groups. ${ }^{\#}=P<0.005$, indicating a significant difference between control and JNK or Akt expressing flies fed only PQ. Error bars represent standard error of the mean. Each data point represents at least three independent replications of 50-60 flies each. (b) The effect of PQ (10 mM) and PQ + MC (10 mM $+1 \mathrm{mM})$ on brains of transgenic lines shown in the images. The overexpression of wild type Akt and Bsk/JNK in dopaminergic neurons provides protection to DA neurons against PQ, however; addition of MC in fails to provide additional protective to transgenic lines, TH-Gal4; UAS-eGFP/UAS-Akt ${ }^{W T}$ and TH-GAL4; $U A S$-eGFP/UAS-bsk $k^{W T}$ against PQ. (c) The average number of neurons per subset was determined $24 \mathrm{hr}$ after the initiation of feeding in these transgenic lines. MC ingestion significantly improved the survival of DA neurons against PQ-induced loss of DA neurons in TH-Gal4; $U A S-e G F P /+$ brains. The overexpression of wild type Akt and JNK supports the survival of DA neurons against $10 \mathrm{mM}$ PQ but addition of $1 \mathrm{mM}$ MC failed to provide additional protection against PQ mediated DA neuron loss in these transgenic lines. ${ }^{* *}=P<0.005$ and represent significant differences between PQ and PQ and MC-treated TH-Gal4; UAS-eGFP/+ brains. " represents significant differences between PQ-treated TH-Gal4; UAS-eGFP/+ and PQ-treated TH-Gal4; UAS-eGFP/UAS-Akt ${ }^{W T}$ and ${ }^{\Phi}$ represent significant differences between PQtreated TH-Gal4; UAS-eGFP/+ and PQ-treated TH-GAL4; UAS-eGFP/UAS-bsk ${ }^{W T}$ brains, respectively. ${ }^{\# / \Phi}=P<0.05$ while ${ }^{\# \#}=P<0.005$. $n=8-12$ brains for each transgenic lines. NS = not significant.

that were fed PQ (Figure 8(b)) displayed significantly greater DA neuron numbers. However, cofeeding of $1 \mathrm{mM} M C$ with PQ failed to provide neuroprotection above that observed in Akt or JNK overexpression (Figures $8(\mathrm{~b})$ and $8(\mathrm{c})$ ). These results are in line of the survival data where the protective effect of MC on transgenic lines, TH-GAL4;UAS-JNK and TH-GAL4;UAS-Akt/+, was proportionate to those observed with control flies, rather than further enhancing survival. 


\section{Discussion}

4.1. MC Imparts Antioxidant Effects in a PQ-Induced Drosophila Model for Parkinson's Disease. PQ is considered an oxidative stressor, generating superoxide and hydroxyl radicals [31]. Moreover, epidemiological and experimental studies point to PQ as an etiological agent for PD [44]. We have used PQ ingestion to establish an in vivo Drosophila PD model [21] and here employ this model to explore genetic interactions that alter the effects of MC, as a model for analysis of drugs offering therapeutic benefits in PD. As may be seen in the results reported in this study, this system has provided a fruitful avenue for exploring the effects of genetic alterations that may play roles in DA neuron susceptibility to deleterious environmental insult. It is noteworthy that we observe strong effects, both on PQ toxicity and on the protective capacity of MC, despite the fact that in all instances we are working with homozygous lethal mutant alleles, necessitating the use of heterozygotes with a wild type allele also present in each instance. It is increasingly recognized that dopaminergic neurons are at heightened oxidative risk, largely due to the interactive nature of DA itself. It is imperative that DA homeostatic mechanisms and oxidative surveillance are sensitively regulated and balanced to avoid catastrophic neuronal destruction. We note that comparable studies in mammalian models, incorporating a large collection of mutant alleles, are more difficult to implement. However, such studies are straightforward using the Drosophila model system, and our results with these heterozygous strains clearly support to prevailing view of the exquisite balance of pathways needed for the well-being of dopaminergic neurons.

MC has been shown to have anti-inflammatory and antioxidant properties in numerous neurodegenerative and injury-induced mammalian models $[15,45]$. Although the exact biological targets for MC are still not well known, it has been reported that MC causes the inhibition of the cytochrome $c$ release from mitochondria, the inhibition of caspase- 1 and -3 expression, and the suppression of microglial activation $[8,15,46]$.

Despite numerous reports supporting the efficacious role of MC in numerous models of neurological disease and injury, other studies have reported the absence of effects or even increased deleterious effects [12, 13]. Diguet et al. [12] reported that the protective or deleterious effects of $\mathrm{MC}$ depend on the mode of administration and dose of the drug. Because there is continuing controversy in MC studies, we first conducted a toxicity test employing a range of $\mathrm{MC}$ doses. Since we found that concentrations above $5 \mathrm{mM}$ were toxic when fed to wild type adult flies, we employed $1 \mathrm{mM}$ $\mathrm{MC}$, a concentration substantially below toxic levels and yet effective in ameliorating the deleterious effects of $10 \mathrm{mM}$ PQ. In addition, we tested various treatment paradigms and found that both pre-feeding and co-feeding regimens were equally protective. Bonilla et al. [19] also reported the prevention of PQ-induced reduction of survival duration in Drosophila, but the mechanism through which MC imparts this effect in Drosophila was not investigated in that study. More recently, Faust et al. [20] tested the efficacy of MC in ameliorating dopaminergic neuron loss in Drosophila when expression of DJ-1A was blocked by specific expression of $D J-1 A R N A i$ in these neurons. In this study of a genetic model for PD, higher concentrations of MC (50-100 mM) were able to rescue the DJ-1RNAi-mediated loss of DA neurons. These investigators also assessed the effect of MC on DA pools and on the survival of DA neurons in the DCM region of the brain. Our study supports and extends this interesting study, demonstrating that this antibiotic is also effective in an environmental toxin model of PD. We also find improvement in survival and DA pools, but also a striking rescue of DA neurons, not only in the specific region investigated by Faust et al. but, interestingly, in all subsets of DA neurons. Mobility also was rescued in our study, as were markers of oxidative stress and inflammation. Thus, the efficacious effects of MC in both a genetic and a toxin model of PD in Drosophila produce comparable outcomes despite the fact that the DJ-1 knockdown effects were observed at approximately 10 to 25 days after eclosion, while this PQ study tested more acute responses. Formally, there is a possibility that MC chemically interacts with and detoxifies PQ. However, the fact that comparable results are observed in both genetic and PQ models of PD in Drosophila argues strongly against this selective chemical interaction. Thus, we conclude that Drosophila systems present a robust model for investigating mechanisms of therapeutic action.

We have shown previously that PQ induces first a rapid activation of DA synthesis, as well as the $\mathrm{BH}_{4}$ cofactor pathway required for both DA biosynthesis and NOS activity, followed by rapid oxidative turnover of $\mathrm{BH}_{4}$ and DA [21]. We therefore expanded our neurochemical analysis to monitor L-DOPA and DOPAC. We detected suppression of each of these neurochemical responses upon MC treatment, confirming a strong antioxidant function for $\mathrm{MC}$ in this system from the perspective of DA metabolite responses as well as for the generation of ROS responses, by catalase and lipid peroxidation assays.

Kraus et al. [11] compared the antioxidant property of MC with other known antioxidants. The antioxidant property was assessed on mammalian neuron cell culture via cellbased glutamate-induced oxidative stress assays and cell-free antioxidant activity assays including lipid peroxidation. MC along with other known antioxidants such as $( \pm)$ tocopherol showed direct radical scavenging activity, proposed to be due to the presence of phenolic ring capable of reacting with free radicals, leading to the formation of relatively stable and unreactive phenol-derived free radicals [15]. Our results, combined with those of Bonilla et al. [19] and Faust et al. [20], strongly support a similar ability of MC in these whole organism studies under a variety of oxidative stress paradigms.

4.2. Failure of MC Protection against PQ in Punch Mutants. Having previously shown that mutants with defects in DA biosynthesis pathways show differential susceptibility to PQ [21], we employed these mutants to test for geneenvironment interactions that might modify the efficacy of 
MC. Interestingly, MC improved the survival of pale and Catsup mutants proportionate to those with wild type flies, while it failed to rescue $P u$ mutants. While one possible explanation for this lack of effect is that a deleterious MC effect is revealed in the $P u$ mutant, we have observed no evidence of altered survival when MC alone, at concentrations employed in this study, is ingested by this mutant strain. These results suggested that functions of $P u$ other than its role in DA homeostasis might impact its effect on $\mathrm{MC}$ protection. $\mathrm{BH}_{4}$, the terminal product of the GTPCH pathway, also functions as an essential cofactor for NO production, facilitating the dimerization of NOS and functioning as a single electron donor during the process of $\mathrm{NO}$ production [35]. The limiting $\mathrm{BH}_{4}$ pools, as in heterozygous $\mathrm{Pu}$ mutants, cause the uncoupling of electron transfer in NOS catalytic function, in consequence, the generation of superoxide and peroxynitrites radicals [35, 47]. In support of this interpretation of our results, we found that Punch mutants exhibited lower than normal NOS activity and that in vivo inhibition of NOS catalytic function with the inhibitor LNAME improved the survival of PQ-fed flies. The inability of MC to prevent excessive generation of peroxynitrites in Punch mutants presents an example of modified drug-gene interaction and may help to explain the phenomenon of drug failure response in PD and other neurodegenerative diseases. Our data also validates Drosophila as an in vivo model for screening of drug molecules for possible drug-gene interaction.

\subsection{Identification of Signal Transduction Pathways Necessary} for MC Protection against PQ-Induced Neurotoxic and Neuroinflammatory Responses. Our investigation of signaling pathways mediating PQ-induced neurotoxic in Drosophila seeks insights into signal transduction pathways that can modify PQ-mediated toxicity at the whole organism level. Most of the previous mammalian studies have utilized in vitro (i.e., cell culture) approaches to address this question. Moreover, these mammalian studies have used primarily pharmacological inhibitors to block the proposed functions of the signaling pathway, which may lack complete specificity of function. Finally, the variations in the type of inhibitor used, inhibitor concentrations, and time of addition as well as cell lines may affect the outcome of these experiments $[48,49]$.

We have tested the roles of kinases and proapoptotic genes known to be functionally conserved with mammals. The heterozygous loss-of-function mutants for JNK/bsk $\left(b s k^{j 27}, b s k^{1}\right.$ and $\left.b s k^{2}\right)$ and $A k t\left(A k t^{1}, A k t^{11627}\right.$ and $\left.A k t^{19894}\right)$ exhibit sensitivity to PQ in the absence of $\mathrm{MC}$ and fail to respond to antibiotic treatment. Moreover, overexpression of the wild type form of either kinase results in resistance to PQ. These results indicate crucial roles for these signaling pathways against neuronal stress responses to PQ. In contrast, heterozygous loss-of-function mutants for ERK and reaper mutants were equivalent to wild type flies in their sensitivity to $\mathrm{PQ}$. We note that this lack of effects does not necessarily indicate an absence of roles for these latter genes. Leaky mutations or redundant functions could easily prevent the detection of deleterious effects. Ongoing studies will address these issues more fully.

In mammals, Akt1 plays a crucial role in cell survival and also is regulated by the PI3K-mediated signaling pathway [41]. Deregulation of the Akt1-mediated signaling pathway has been well documented in familial and sporadic forms of PD models $[38,50]$. Stimulation of the Akt1 signaling pathway in in vitro or in vivo models resulted in neurotrophic, antiapoptotic effects [41]. Yang et al. [50] found suppression of ROS and survival of DA neurons in transgenic strains over-expressing Drosophila Akt1 in DJ1 RNAi strains, again illustrating that the PQ model parallels genetic PD models in many respects.

In mammalian PD models, JNK, which initiates programmed cell death by inactivating the antiapoptotic protein $\mathrm{Bcl}-\mathrm{xl}$, is activated in $\mathrm{TH}$ neurons in a PQ-induced mammalian PD model suggesting a role JNK in neurodegeneration [51]. Similarly, activated JNK has been detected in Parkin mutants in Drosophila [52]. SP600125, a specific JNK inhibitor cofed with $20 \mathrm{mM}$ PQ increased the survival and locomotory activity when compared with those fed with $20 \mathrm{mM}$ PQ [53]. Moreover, Wang et al. [54] also demonstrated important roles for JNK in longevity and resistance to PQ-induced oxidative stress. Our data suggest a specific role of JNK in the survival response of DA neurons in our PD model. We further confirm the prosurvival role of $A k t 1$ and JNK in DA neurons against PQ with evaluation of delayed DA neuron loss with overexpression of wild type Akt and JNK in DA neurons. However, addition of MC failed to further prevent the DA neuron loss in the Akt over-expressed DA neurons against PQ. These neuron count data parallel the survival data obtained with the flies over-expressed with wild type Akt and JNK against PQ and PQ with MC. MC improved the survival of the flies over-expressed with wild type Akt and JNK in the same proportional as in wild type (control) flies against PQ.

We found that heterozygous loss-of-function mutant ERK/rolled lacks any detectable functional involvement in our model, although the result could also infer the lack of sufficient knockdown due to the heterozygosity of the strain. Recently, genetic interaction between Drosophila DJ-1 and Ras/ERK but not with PI3K/Akt thereby indicating that the prosurvival effect of DJ-1 is mediated via ERK in DA neurons [55]. While the lack of effect in our examination of an ERK/rolled mutant may be explained, as noted above, by insufficient reduction of function, this may also be an instance in which the cellular effects of PQ and mutations in PD genes may diverge. Future studies will address this point.

Finally, we analyzed the role of programmed cell death in PQ neurotoxicity by testing heterozygous mutants for the pro-apoptotic gene, reaper, and the programmed cell death initiator, caspase- 9 ortholog, dronc. We found that a heterozygous loss-of-function reaper mutant displayed comparable survival to wild type flies on $\mathrm{PQ}$, while dronc mutants showed an increase in lifespan. PQ has been shown to induce apoptosis via activation of caspases 3 and 9 and inhibition of Bcl-2 family members except Bax [56]. Thus, our results for dronc suggest a parallel mode of action in flies and mammals. 
In conclusion, in addition to our finding of protective mechanisms of MC against PQ in this PD model and novel MC-Punch mutant interactions, our results here provide in vivo evidence for essential roles for stress-responsive kinases in the response to $\mathrm{PQ}$. In this paper, we present data showing the failure of heterozygous Akt1 and JNK loss-offunction mutants to respond to MC. However, ingestion of $10 \mathrm{mM} \mathrm{MC}$ by flies over-expressing wild type Akt1 and JNK in DA neurons does not provide additional levels of protection against $\mathrm{PQ}$. Thus, these pathways may not be directly affected by $\mathrm{MC}$ or, alternatively, there may be an upper limit above which these kinases can no longer be effective as damage from continuous PQ exposure in these acute toxin model accumulates. Further exploration of the roles of these pathways in various mutant backgrounds in chronic or sporadic exposure models of the effects of PQ will be productive avenues to follow as will analyses of tissuespecific Akt and JNK responses.

\section{Acknowledgments}

This work was supported in part by grants from the American Parkinson Disease Foundation and the National Institutes of Health (R15ND078728) to J. O'Donnell. In addition, the authors gratefully acknowledge additional support from the University of Alabama. They wish to thank Jay Hirsh and Tien Hsu for Drosophila strains and other members of the O'Donnell lab for their insights and suggestions for this project. All HPLC analyses were expertly performed by research assistant Janna Brown.

\section{References}

[1] R. Betarbet, T. B. Sherer, G. MacKenzie, M. Garcia-Osuna, A. V. Panov, and J. T. Greenamyre, "Chronic systemic pesticide exposure reproduces features of Parkinson's disease," Nature Neuroscience, vol. 3, no. 12, pp. 1301-1306, 2000.

[2] A. L. McCormack, J. G. Atienza, L. C. Johnston, J. K. Andersen, $\mathrm{S}$. $\mathrm{Vu}$, and D. A. Di Monte, "Role of oxidative stress in paraquat-induced dopaminergic cell degeneration," Journal of Neurochemistry, vol. 93, no. 4, pp. 1030-1037, 2005.

[3] K. Nuytemans, J. Theuns, M. Cruts, and C. van Broeckhoven, "Genetic etiology of Parkinson disease associated with mutations in the SNCA, PARK2, PINK1, PARK7, and LRRK2 genes: a mutation update," Human Mutation, vol. 31, no. 7, pp. 763780, 2010.

[4] S. Hunot, F. Boissière, B. Faucheux et al., "Nitric oxide synthase and neuronal vulnerability in Parkinson's disease," Neuroscience, vol. 72, no. 2, pp. 355-363, 1996.

[5] C. K. Glass, K. Saijo, B. Winner, M. C. Marchetto, and F. H. Gage, "Mechanisms underlying inflammation in neurodegeneration," Cell, vol. 140, no. 6, pp. 918-934, 2010.

[6] H. Macdonald, R. G. Kelly, E. S. Allen, J. F. Noble, and L. A. Kanegis, "Pharmacokinetic studies on minocycline in man," Clinical Pharmacology and Therapeutics, vol. 14, no. 5, pp. 852-861, 1973.

[7] Y. Du, Z. Ma, S. Lin et al., "Minocycline prevents nigrostriatal dopaminergic neurodegeneration in the MPTP model of Parkinson's disease," Proceedings of the National Academy of Sciences of the United States of America, vol. 98, no. 25, pp. 14669-14674, 2001.
[8] D. C. Wu, V. Jackson-Lewis, M. Vila et al., "Blockade of microglial activation is neuroprotective in the 1-methyl-4phenyl-1,2,3,6-tetrahydropyridine mouse model of Parkinson disease," Journal of Neuroscience, vol. 22, no. 5, pp. 1763-1771, 2002.

[9] X. Wang, S. Zhu, M. Drozda et al., "Minocycline inhibits caspase-independent and -dependent mitochondrial cell death pathways in models of Huntington's disease," Proceedings of the National Academy of Sciences of the United States of America, vol. 100, no. 18, pp. 10483-10487, 2003.

[10] M. G. Purisai, A. L. McCormack, S. Cumine, J. Li, M. Z. Isla, and D. A. Di Monte, "Microglial activation as a priming event leading to paraquat-induced dopaminergic cell degeneration," Neurobiology of Disease, vol. 25, no. 2, pp. 392-400, 2007.

[11] R. L. Kraus, R. Pasieczny, K. Lariosa-Willingham, M. S. Turner, A. Jiang, and J. W. Trauger, "Antioxidant properties of minocycline: neuroprotection in an oxidative stress assay and direct radical-scavenging activity," Journal of Neurochemistry, vol. 94, no. 3, pp. 819-827, 2005.

[12] E. Diguet, P. O. Fernagut, X. Wei et al., "Deleterious effects of minocycline in animal models of Parkinson's disease and Huntington's disease," European Journal of Neuroscience, vol. 19, no. 12, pp. 3266-3276, 2004.

[13] M. Tsuji, M. A. Wilson, M. S. Lange, and M. V. Johnston, "Minocycline worsens hypoxic-ischemic brain injury in a neonatal mouse model," Experimental Neurology, vol. 189, no. 1, pp. 58-65, 2004.

[14] K. Kupsch, S. Hertel, P. Kreutzmann et al., "Impairment of mitochondrial function by minocycline," FEBS Journal, vol. 276, no. 6, pp. 1729-1738, 2009.

[15] J. M. Plane, Y. Shen, D. E. Pleasure, and W. Deng, "Prospects for minocycline neuroprotection," Archives of Neurology, vol. 67, no. 12, pp. 1442-1448, 2010.

[16] D. M. Roden and A. L. George, "The genetic basis of variability in drug responses," Nature Reviews Drug Discovery, vol. 1, no. 1, pp. 37-44, 2002.

[17] S. S. Ambegaokar, B. Roy, and G. R. Jackson, "Neurodegenerative models in Drosophila: Polyglutamine disorders, Parkinson disease, and amyotrophic lateral sclerosis," Neurobiology of Disease, vol. 40, no. 1, pp. 29-39, 2010.

[18] I. S. Pienaar, J. Götz, and M. B. Feany, "Parkinson's disease: insights from non-traditional model organisms," Progress in Neurobiology, vol. 92, no. 4, pp. 558-571, 2010.

[19] E. Bonilla, S. Medina-Leendertz, V. Villalobos, L. Molero, and A. Bohórquez, "Paraquat-induced oxidative stress in Drosophila melanogaster: effects of melatonin, glutathione, serotonin, minocycline, lipoic acid and ascorbic acid," Neurochemical Research, vol. 31, no. 12, pp. 1425-1432, 2006.

[20] K. Faust, S. Gehrke, Y. Yang, L. Yang, M. F. Beal, and B. $\mathrm{Lu}$, "Neuroprotective effects of compounds with antioxidant and anti-inflammatory properties in a Drosophila model of Parkinson's disease," BMC Neuroscience, vol. 10, article 109, 2009.

[21] A. Chaudhuri, K. Bowling, C. Funderburk et al., "Interaction of genetic and environmental factors in a Drosophila parkinsonism model," Journal of Neuroscience, vol. 27, no. 10, pp. 2457-2467, 2007.

[22] Z. Wang, F. Ferdousy, H. Lawal et al., "Catecholamines up integrates dopamine synthesis and synaptic trafficking," Journal of Neurochemistry, vol. 119, no. 6, pp. 1294-1305, 2011.

[23] F. Friggi-Grelin, H. Coulom, M. Meller, D. Gomez, J. Hirsh, and S. Birman, "Targeted gene expression in Drosophila dopaminergic cells using regulatory sequences from tyrosine 
hydroxylase," Journal of Neurobiology, vol. 54, no. 4, pp. 618627, 2003.

[24] D. G. Stathakis, D. Y. Burton, W. E. McIvor, S. Krishnakumar, T. R. F. Wright, and J. M. O'Donnell, “The catecholamines up (Catsup) protein of Drosophila melanogaster functions as a negative regulator of tyrosine hydroxylase activity," Genetics, vol. 153, no. 5, pp. 361-382, 1999.

[25] W. S. Neckameyer and K. White, "Drosophila tyrosine hydroxylase is encoded by the pale locus," Journal of Neurogenetics, vol. 8, no. 4, pp. 189-199, 1993.

[26] W. J. Mackay, E. R. Reynolds, and J. M. O’Donnell, “Tissuespecific and complex complementation patterns in the Punch locus of Drosophila melanogaster," Genetics, vol. 111, no. 4, pp. 885-904, 1985.

[27] C. McClung and J. Hirsh, "The trace amine tyramine is essential for sensitization to cocaine in Drosophila," Current Biology, vol. 9, no. 16, pp. 853-860, 1999.

[28] G. C. Bewley, J. A. Nahmias, and J. L. Cook, "Developmental and tissue-specific control of catalase expression in Drosophila melanogaster: correlations with rates of enzyme synthesis and degradation," Developmental Genetics, vol. 4, no. 1, pp. 49-60, 1983.

[29] J. A. Buege and S. D. Aust, "Microsomal lipid peroxidation," Methods in Enzymology, vol. 52, pp. 302-310, 1978.

[30] J. Kriz, M. D. Nguyen, and J. P. Julien, "Minocycline slows disease progression in a mouse model of amyotrophic lateral sclerosis," Neurobiology of Disease, vol. 10, no. 3, pp. 268-278, 2002.

[31] S. Zhu, I. G. Stavrovskaya, M. Drozda et al., "Minocycline inhibits cytochrome $c$ release and delays progression of amyotrophic lateral sclerosis in mice," Nature, vol. 417, no. 6884, pp. 74-78, 2002.

[32] J. S. Bus, S. D. Aust, and J. E. Gibson, "Superoxide and singlet oxygen catalyzed lipid peroxidation as a possible mechanism for paraquat (methyl viologen) toxicity," Biochemical and Biophysical Research Communications, vol. 58, no. 3, pp. 749755,1974

[33] S. Krishnakumar, D. Burton, J. Rasco, X. Chen, and J. O'Donnell, "Functional interactions between GTP cyclohydrolase I and tyrosine hydroxylase in Drosophila," Journal of Neurogenetics, vol. 14, no. 1, pp. 1-23, 2000.

[34] A. Presta, U. Siddhanta, C. Wu et al., "Comparative functioning of dihydro- and tetrahydropterins in supporting electron transfer, catalysis, and subunit dimerization in inducible nitric oxide synthase," Biochemistry, vol. 37, no. 1, pp. 298-310, 1998.

[35] R. H. Foxton, J. M. Land, and S. J. R. Heales, "Tetrahydrobiopterin availability in Parkinson's and Alzheimer's disease; potential pathogenic mechanisms," Neurochemical Research, vol. 32, no. 4-5, pp. 751-756, 2007.

[36] K. Toska, R. Kleppe, C. G. Armstrong, N. A. Morrice, P. Cohen, and J. Haavik, "Regulation of tyrosine hydroxylase by stressactivated protein kinases," Journal of Neurochemistry, vol. 83, no. 4, pp. 775-783, 2002.

[37] W. S. Neckameyer and J. S. Weinstein, "Stress affects dopaminergic signaling pathways in Drosophila melanogaster," Stress, vol. 8, no. 2, pp. 117-131, 2005.

[38] H. Dudek, S. R. Datta, T. F. Franke et al., "Regulation of neuronal survival by the serine-threonine protein kinase Akt," Science, vol. 275, no. 5300, pp. 661-665, 1997.

[39] C. T. Chu, D. J. Levinthal, S. M. Kulich, E. M. Chalovich, and D. B. DeFranco, "Oxidative neuronal injury: the dark side of
ERK1/2," European Journal of Biochemistry, vol. 271, no. 11, pp. 2060-2066, 2004.

[40] L. Chang and M. Karin, "Mammalian MAP kinase signalling cascades," Nature, vol. 410, no. 6824, pp. 37-40, 2001.

[41] R. E. Burke, "Inhibition of mitogen-activated protein kinase and stimulation of Akt kinase signaling pathways: two approaches with therapeutic potential in the treatment of neurodegenerative disease," Pharmacology and Therapeutics, vol. 114, no. 3, pp. 261-277, 2007.

[42] S. J. Harper and P. LoGrasso, "Signalling for survival and death in neurones: the role of stress-activated kinases, JNK and p38," Cellular Signalling, vol. 13, no. 5, pp. 299-310, 2001.

[43] A. H. Brand and N. Perrimon, "Targeted gene expression as a means of altering cell fates and generating dominant phenotypes," Development, vol. 118, no. 2, pp. 401-415, 1993.

[44] R. J. Dinis-Oliveira, F. Remião, H. Carmo et al., "Paraquat exposure as an etiological factor of Parkinson's disease," NeuroToxicology, vol. 27, no. 6, pp. 1110-1122, 2006.

[45] H. S. Kim and Y. H. Suh, "Minocycline and neurodegenerative diseases," Behavioural Brain Research, vol. 196, no. 2, pp. 168179, 2009.

[46] M. Chen, V. O. Ona, M. Li et al., "Minocycline inhibits caspase- 1 and caspase- 3 expression and delays mortality in a transgenic mouse model of Huntington disease," Nature Medicine, vol. 6, no. 7, pp. 797-801, 2000.

[47] E. R. Werner, A. C. F. Gorren, R. Heller, G. Werner-Felmayer, and B. Mayer, "Tetrahydrobiopterin and nitric oxide: mechanistic and pharmacological aspects," Experimental Biology and Medicine, vol. 228, no. 11, pp. 1291-1302, 2003.

[48] K. Sekiguchi, T. Yokoyama, M. Kurabayashi, F. Okajima, and R. Nagai, "Sphingosylphosphorylcholine induces a hypertrophic growth response through the mitogen-activated protein kinase signaling cascade in rat neonatal cardiac myocytes," Circulation Research, vol. 85, no. 11, pp. 1000-1008, 1999.

[49] V. Waetzig and T. Herdegen, "MEKK1 controls neurite regrowth after experimental injury by balancing ERK1/2 and JNK2 signaling," Molecular and Cellular Neuroscience, vol. 30, no. 1, pp. 67-78, 2005.

[50] Y. Yang, S. Gehrke, M. E. Haque et al., "Inactivation of Drosophila DJ-1 leads to impairments of oxidative stress response and phosphatidylinositol 3-kinase/Akt signaling," Proceedings of the National Academy of Sciences of the United States of America, vol. 102, no. 38, pp. 13670-13675, 2005.

[51] J. Peng, X. O. Mao, F. F. Stevenson, M. Hsu, and J. K. Andersen, "The herbicide paraquat induces dopaminergic nigral apoptosis through sustained activation of the JNK pathway," Journal of Biological Chemistry, vol. 279, no. 31, pp. 32626-32632, 2004.

[52] G. H. Cha, S. Kim, J. Park et al., "Parkin negatively regulates JNK pathway in the dopaminergic neurons of Drosophila," Proceedings of the National Academy of Sciences of the United States of America, vol. 102, no. 29, pp. 10345-10350, 2005.

[53] M. Jimenez-Del-Rio, A. Daza-Restrepo, and C. VelezPardo, "The cannabinoid CP55,940 prolongs survival and improves locomotor activity in Drosophila melanogaster against paraquat: implications in Parkinson's disease," Neuroscience Research, vol. 61, no. 4, pp. 404-411, 2008.

[54] M. C. Wang, D. Bohmann, and H. Jasper, "JNK signaling confers tolerance to oxidative stress and extends lifespan in Drosophila," Developmental Cell, vol. 5, no. 5, pp. 811-816, 2003. 
[55] L. Aron, P. Klein, T. T. Pham, E. R. Kramer, W. Wurst, and R. Klein, "Pro-survival role for Parkinson's associated gene DJ-1 revealed in trophically impaired dopaminergic neurons," PLoS Biology, vol. 8, no. 4, Article ID e1000349, 2010.

[56] Q. Fei, A. L. McCormack, D. A. Di Monte, and D. W. Ethell, "Paraquat neurotoxicity is mediated by a Bak-dependent mechanism," Journal of Biological Chemistry, vol. 283, no. 6, pp. 3357-3364, 2008. 


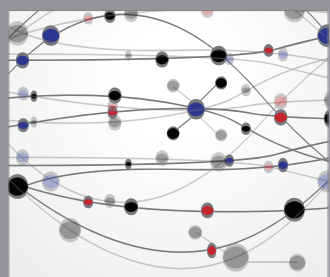

The Scientific World Journal
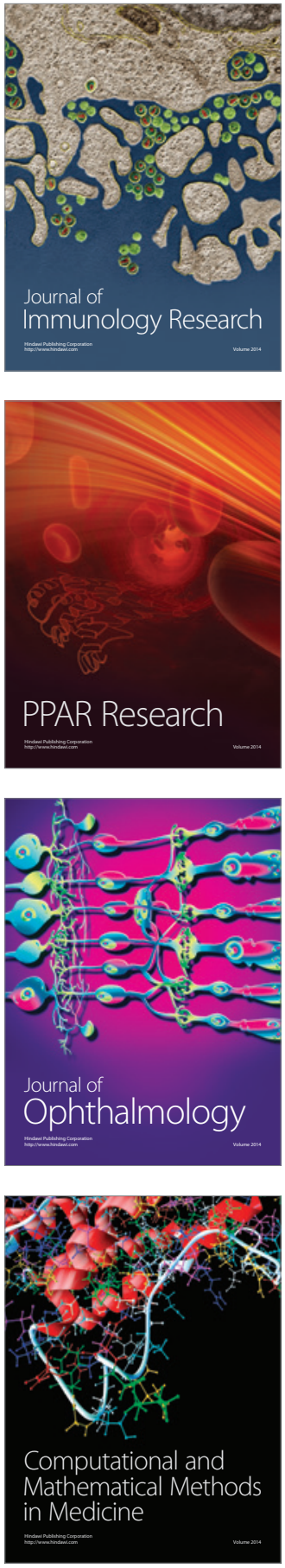

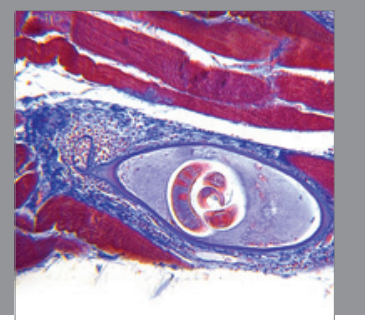

Gastroenterology

Research and Practice
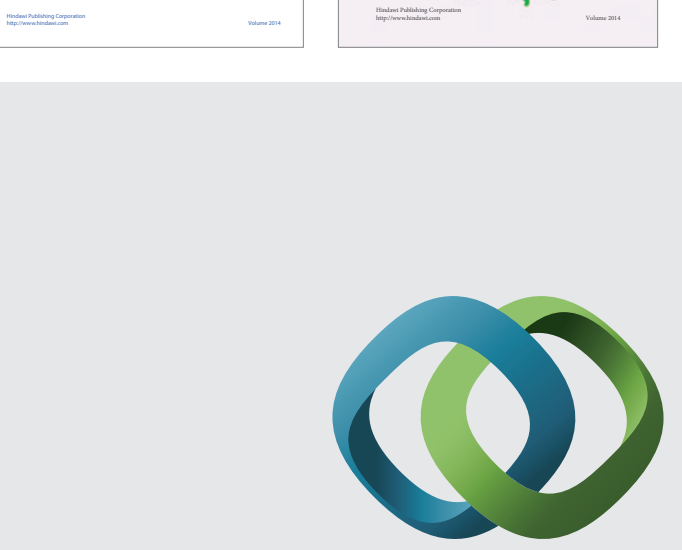

\section{Hindawi}

Submit your manuscripts at

http://www.hindawi.com
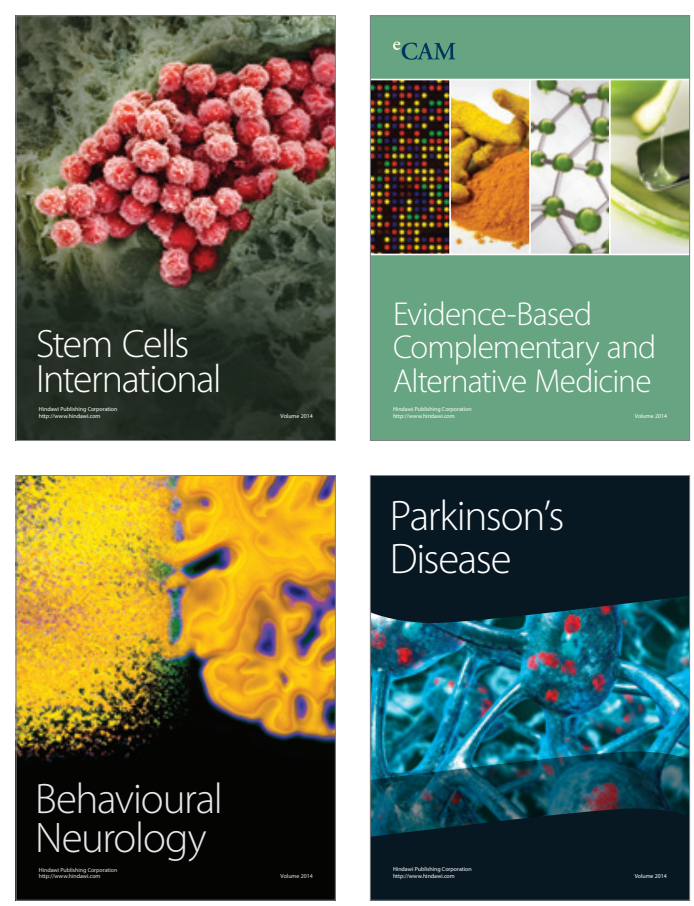

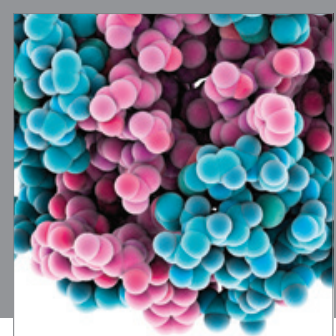

Journal of
Diabetes Research

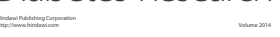

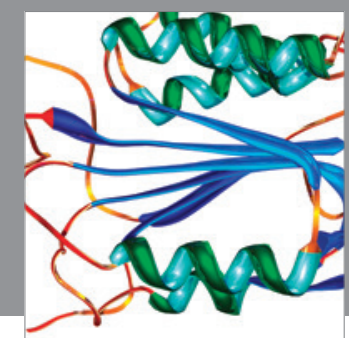

Disease Markers
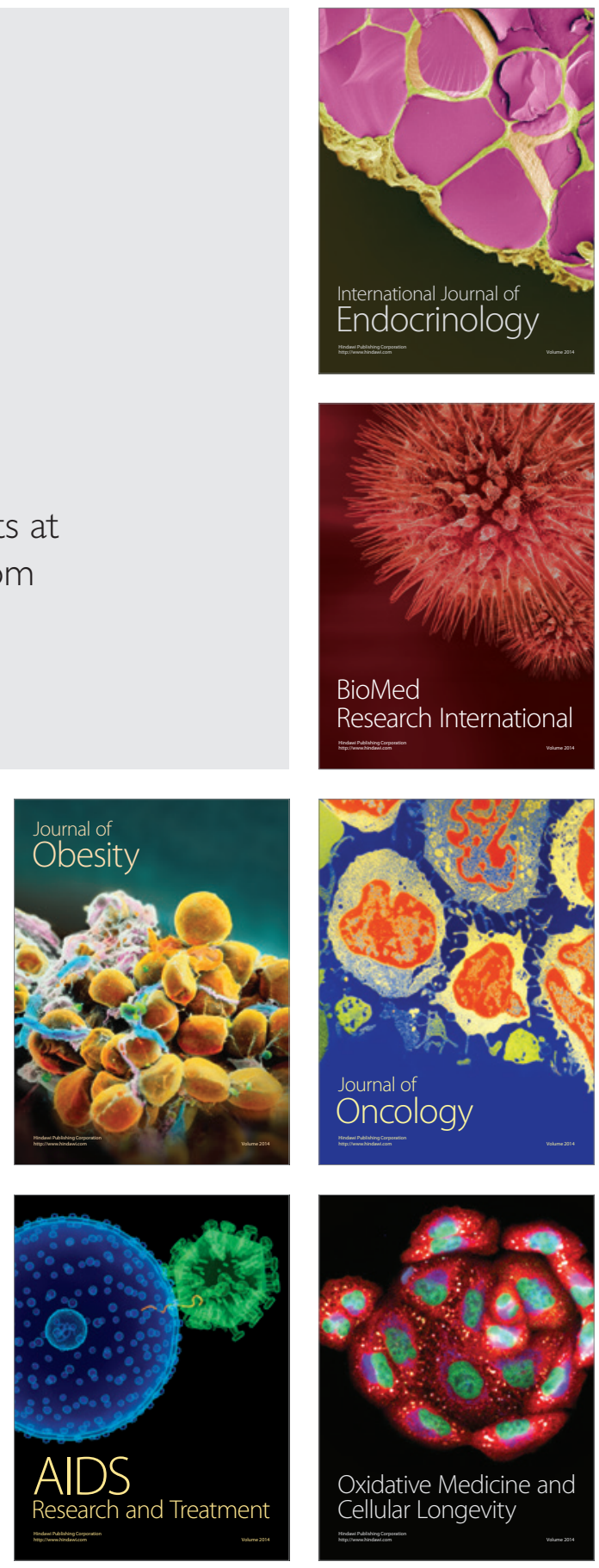